\title{
Dislocation transport using a time-explicit Runge-Kutta discontinuous Galerkin finite element approach
}

\author{
Manas V. Upadhyay ${ }^{1, *}$, Jérémy Bleyer ${ }^{2}$ \\ ${ }^{1}$ Laboratoire de Mécanique des Solides (LMS), CNRS UMR 7649, Ecole \\ Polytechnique, Institut Polytechnique de Paris, Route de Saclay, 91128 Palaiseau \\ Cedex, France \\ ${ }^{2}$ Laboratoire Navier, CNRS UMR 8205, Ecole des Ponts ParisTech, Université \\ Gustave Eiffel, 6 et 8 avenue Blaise Pascal, 77455 Marne-la-Vallée Cedex 2, France \\ E-mail: manas.upadhyay@polytechnique.edu (corresponding author*)
}

\begin{abstract}
.
A time-explicit Runge-Kutta discontinuous Galerkin (RKDG) finite element scheme is proposed to solve the dislocation transport initial boundary value problem in 3D. The dislocation density transport equation, which lies at the core of this problem, is a first-order unsteady-state advection-reaction-type hyperbolic partial differential equation; the DG approach is well suited to solve such equations that lack any diffusion terms. The development of the RKDG scheme follows the method of lines approach. First, a space semi-discretization is performed using the DG approach with upwinding to obtain a system of ordinary differential equations in time. Then, time discretization is performed using explicit RK schemes to solve this system. The 3D numerical implementation of the RKDG scheme is performed for the first-order (forward Euler), second-order and third-order RK methods using the strong stability preserving approach. These implementations provide (quasi-)optimal convergence rates for smooth solutions. A slope limiter is used to prevent spurious Gibbs oscillations arising from high-order space approximations (polynomial degree $\geq 1$ ) of rough solutions. A parametric study is performed to understand the influence of key parameters of the RKDG scheme on the stability of the solution predicted during a screw dislocation transport simulation. Then, annihilation of two oppositely signed screw dislocations and the expansion of a polygonal dislocation loop are simulated. The RKDG scheme is able to resolve the shock generated during dislocation annihilation without any spurious oscillations and predict the prismatic loop expansion with very low numerical diffusion. These results demonstrate the robustness of the scheme.
\end{abstract}

Keywords: Discontinuous Galerkin, Runge Kutta, finite elements, FEM, dislocation, transport, hyperbolic equations

Submitted to: Modelling Simul. Mater. Sci. Eng. 


\section{Introduction}

Dislocations are line-type defects occurring in crystalline materials. They are characterized by (i) a Burgers vector, which is obtained from the closure failure of a Burgers circuit drawn around a dislocation in a dislocated crystal [1, 2], and (ii) their line direction. In a continuum framework, they can be represented either as discrete (singular) lines or as fields (finite volumes). The kinematics of dislocation fields has been a subject of interest since the work of Nye [3], who characterized dislocations using a second-order polar dislocation density tensor defined as the outer product of the Burgers vector and the dislocation line vector. The main equation in the kinematic theory of dislocation fields is the dislocation transport equation, which is a first-order unsteadystate advection-reaction-type hyperbolic partial differential equation of the evolution of the polar dislocation density tensor; it is obtained from the conservation of the Burgers vector of evolving dislocations [4]. Along with this equation, the dislocation continuity condition, dislocation in-flux and zero dislocation out-flux boundary conditions, and the initial configuration of dislocations, constitute the dislocation transport initial boundary value problem (IBVP). This problem can be solved to predict dislocation motion, annihilation of two oppositely signed dislocations and expansion/contraction of dislocation loops without using additional rules in the system of equations. The dislocation transport IBVP has been coupled with the theory of lattice incompatibility and internal stresses within dislocated crystals in an isothermal framework to obtain the dynamic field dislocation mechanics model [5, 6]. More recently, the dynamic thermal field dislocation mechanics model [7] was proposed to account for a coupling between the field dislocation mechanics and the heat transfer problem.

A plethora of numerical techniques exist to solve hyperbolic partial differential equations including those based on the finite element (FE) approach and the fast Fourier transform approach. All these approaches involve discretizing the continuous domain into a discrete (finite) set of elements or points where the solution is sought. This space discretization induces roughness in the solution even if the initial configuration in the continuous domain is smooth. In addition, discontinuities can be introduced in the solution, for example, when two oppositely signed dislocations interact and annihilate. In general, the presence of roughness in the solution can result in highly oscillatory and incorrect approximations when standard continuous Galerkin (CG) FE method [8] or spectral methods [9] are used. The origin of these errors is that unlike elliptic or parabolic partial differential equations, there is no physical diffusion term present in purely hyperbolic partial differential equations. One could envisage using the classical artificial diffusion scheme (also known as the viscosity method) or a Least-Squares (LS) approach to avoid such oscillations, however, the resulting solution is often highly damped [8].

One way to overcome these problems is to use hybrid approaches to obtain a tradeoff between highly oscillatory and highly diffuse solutions. Thus far, the dislocation transport problem has been solved using the following hybrid approaches: (i) weighted 
CG least squares (LS) FE scheme [10, 11, 12] with a streamline diffusion term [13] in the non-linear case, and (ii) the fast Fourier transform approach with spectral filters [14]. Another approach involves solving the dislocation transport problem in two steps. The first step focuses on solving only the flux term (related to the plastic distortion rate [6]) in the dislocation transport equation by representing it as a system of Hamilton-Jacobitype non-linear hyperbolic equations. These equations have been numerically solved in a 1-dimensional (1D) and 2-dimensional (2D) setting using a Godunov-type high resolution scheme [15, 16]; a 3-dimensional (3D) extension has not yet been proposed. The second step involves updating the dislocation density rate, which is related to the curl of the plastic distortion rate. Even though this approach does not directly solve the dislocation transport equation, the results show a significant improvement over the existing hybrid approaches [15, 16].

A more natural approach to numerically solve advection-reaction-type hyperbolic partial differential equations is the discontinuous Galerkin (DG) FE approach. The DG approach seeks piecewise-continuous solutions belonging to a piecewise continuous function space, which is better equipped than continuous function spaces used in the CG approach to deal with roughness or discontinuities in the solution. Furthermore, the DG approach is "conservative" in the sense that the dislocation density that "flows" out of a mesh element through one of its faces "flows" into the adjacent element through that face, and this flow can be quantified using a flux term; this property makes the DG approach better suited than a CG approach to solve transport problems. In addition, the mass matrix in the DG approach is block diagonal and its inversion can be massively parallelized resulting in significant computational gains in comparison to a CG approach for the same mesh and order of discretization. Other advantages include the use of a general mesh (in comparison to a simplicial mesh for the CG approach), the use of non-standard shape functions and the ability to solve different governing equations for different elements. However, due to the use of piecewise continuous functions, the DG approach involves using a higher number of degrees of freedom with increased memory and storage space requirements than the CG approach.

With respect to time discretization, thus far only first-order accurate implicit (backward Euler) [10] and first-order accurate explicit (forward Euler) [11, 14] RungeKutta (RK) schemes have been used to solve the dislocation transport equation; note that a second-order accurate explicit (modified Euler/midpoint/Heun's method) RK scheme was used to solve the non-linear Hamilton-Jacobi-type hyperbolic equation for plastic distortion rate evolution [16]. In general, explicit RK schemes are computationally faster than implicit RK schemes, which was the main reason why [11] adopted the forward Euler scheme over the backward Euler scheme of [10]. The time step of an explicit RK scheme has an upper bound typically set by the usual CourantFrederichs-Lewy (CFL) condition [17, 18]. This condition imposes a very stringent restriction on the time step of the forward Euler scheme whenever polynomials of degree greater than or equal to 1 are used for the space approximation. For such problems, using high-order (greater than 1) accurate explicit RK schemes can be better than using 
the forward Euler scheme from the point-of-view of the trade-off between computational time and accuracy of the solution; this was also noted in [16].

In this work, we propose a time-explicit 3-dimensional (3D) RKDG FE approach to solve the dislocation transport IBVP. The development follows the so-called method of lines approach [19, 20]. Starting with the continuous variational formulation, this approach involves first discretizing the spatial derivatives entering the partial differential equation of the formulation. This space semidiscretization transforms the partial differential equation into a system of coupled ordinary differential equations in time. This system is then discretized in time using explicit RK schemes. We have done the 3D numerical implementation of the RKDG method using the Strong Stability Preserving (SSP) approach [21] for three explicit RK schemes of order 1,2 and 3 and compared their predictions. The RKDG approach allows using piecewise-constant functions as well as high-order space approximations (polynomial degree greater than or equal to 1) to approximate the solution. Using high-order space approximation, however, induces spurious oscillations (Gibbs oscillations) whenever some roughness, discontinuities or shocks are present in the solution [9, 22]. Slope limiters can be used to eliminate the spurious oscillations [20]. We use the hierarchical vertex-based slope limiter developed by Kuzmin [23, 24]. Note that Morin et al. [16] used a minmod slope limiter to eliminate the spurious oscillations from their predicted plastic distortion solution.

An implicit RKDG scheme [25, 26, 27] has previously been used to solve the governing equations of the so-called continuum dislocation dynamics theory [28]. This implementation is fundamentally different from the approach proposed in the present work in the following manner. The first difference is in the governing equations of the dislocation transport IBVP of the present work and in the continuum dislocation dynamics theory. There are three governing equations for dislocation evolution in the latter theory: evolution of (i) a scalar dislocation number density, (ii) a dislocation density vector and (iii) a scalar dislocation curvature tensor. These equations are obtained from a reformulation of the second-order tensorial dislocation transport equation by neglecting the temporal evolution of the Burgers vector, which is assumed to be constant for a dislocation line at any given instant in time. While this assumption works well in an isothermal case, however, it may not be applicable in the case of dislocation transport in a transient heterogeneous temperature field [7]. In this regard, it may become necessary to use the original second-order tensorial dislocation transport equation as done in this work. The second difference comes from the manner in which the numerical implementation of these models has been performed. The three equations of the continuous dislocation dynamics theory [25, 26, 27] are implemented in their conservative form, which requires dealing with numerical fluxes across element interfaces and mesh boundaries using the flux term of this form. Each equation has its own flux term and a corresponding numerical flux. The approach adopted in this work requires a single expression of the numerical flux that is directly based on the dislocation density and its velocity, making its physical interpretation rather straightforward. The third difference is the use of explicit RK schemes in the present work and the use of implicit 
RK schemes in [25, 26, 27]; the error estimates, convergence criteria and stability of the numerical schemes are different for both approaches. The fourth difference comes from the use of slope limiters in the present work to eliminate spurious Gibbs oscillations that arise when rough solutions are approximated using high order polynomials.

The remainder of this article is divided into the following sections. The tensorial notations used in this work are presented in section 2. The dislocation transport IBVP is briefly recalled in section 3. The explicit RKDG formulation for the dislocation transport IBVP is proposed in section 4 and its properties are discussed. The 3D numerical implementation of the RKDG scheme using explicit RK schemes of order 1, 2 and 3, together with the CFL condition, slope limiters and the algorithm are presented in section 5. Simulation setup of three model problems, viz. transport of a single screw dislocation, annihilation of two oppositely signed screw dislocations and expansion of a polygonal dislocation loop, and the simulation results are presented and discussed in section 6. The summary, conclusions and some future perspectives are presented in section 7. Definitions specific to the DG implementation are presented in the appendix. Supporting simulation videos and images are provided as supplementary material. 


\section{Notations}

The notations presented below are adopted from the book of Salencon [29]. Scalars (zeroth order tensors) are denoted with regular font. First order tensors, i.e. vectors, are denoted with bold and italic lowercase letters; unit vectors are denoted with an additional overhead hat symbol ^. Second order tensors are denoted with bold and italic uppercase letters or bold and italic Greek symbols; the only exception is the second order identity tensor $\mathbb{I}=\delta_{i j} \hat{e}_{i} \otimes \hat{e}_{j}$, where $\delta_{i j}$ is the Kronecker delta function. Third order tensors are denoted with bold non-italic uppercase letters. Null non-scalar tensorial quantities are represented by equating them to the bold font zero symbol, i.e. 0. Whenever the Einstein notation is used, tensors are represented using a nonbold font. The Einstein summation convention will be implied for operations between tensorial quantities; in this section, it is used to understand different tensorial operations considered in this work.

Consider two vectors $\boldsymbol{a}=a_{i} \hat{e}_{i}$ and $\boldsymbol{b}=b_{i} \hat{e}_{i}$, and two asymmetric second order tensors $\boldsymbol{A}=A_{i j} \hat{e}_{i} \otimes \hat{e}_{j}$ and $\boldsymbol{\beta}=\beta_{i j} \hat{e}_{i} \otimes \hat{e}_{j}$ in the rectangular Cartesian basis with unit vectors $\hat{\boldsymbol{e}}$. In this work, we will use the following operations:

Transposition:

$$
\boldsymbol{A}^{T}=A_{j i} \hat{e}_{i} \otimes \hat{e}_{j}
$$

Tensorial (outer) product:

$$
(\boldsymbol{a} \otimes \boldsymbol{b})_{i j}=a_{i} b_{j} \hat{e}_{i} \otimes \hat{e}_{j}
$$

Inner (dot) product:

$$
\begin{aligned}
& (\boldsymbol{a} \cdot \boldsymbol{b})=(\boldsymbol{b} \cdot \boldsymbol{a})=a_{i} b_{i} \\
& (\boldsymbol{A}: \boldsymbol{\beta})=(\boldsymbol{\beta}: \boldsymbol{A})=A_{i j} \beta_{i j} \\
& (\boldsymbol{A} \cdot \boldsymbol{\beta})_{i k}=A_{i j} \beta_{j k} \hat{e}_{i} \otimes \hat{e}_{k}
\end{aligned}
$$

where ":" is the symbol for a double dot product.

Cross product between a second-order tensor and a vector:

$$
(\boldsymbol{A} \times \boldsymbol{b})_{i j}=e_{j k l} A_{i k} b_{l} \hat{e}_{i} \otimes \hat{e}_{j}
$$

where $e_{i j k}$ is a component of the third order Levi-Civita permutation tensor $\mathbf{X}=$ $e_{i j k} \hat{e}_{i} \otimes \hat{e}_{j} \otimes \hat{e}_{k}$.

Tensorial operations involving vector differential operator $\nabla=\frac{\partial}{\partial_{i}} \hat{e}_{i}$ :

$$
\begin{aligned}
& (\nabla \boldsymbol{a})_{i j}=(\operatorname{grad} \boldsymbol{a})_{i j}=a_{i, j} \hat{e}_{i} \otimes \hat{e}_{j} \\
& (\nabla \boldsymbol{a})_{j i}^{T}=(\operatorname{grad} \boldsymbol{a})_{j i}^{T}=a_{j, i} \hat{e}_{i} \otimes \hat{e}_{j} \\
& (\nabla \boldsymbol{A})_{i j k}=(\operatorname{grad} \boldsymbol{A})_{i j k}=A_{i j, k} \hat{e}_{i} \otimes \hat{e}_{j} \otimes \hat{e}_{k} \\
& (\nabla \cdot \boldsymbol{a})=(\operatorname{div} \boldsymbol{a})=a_{i, i} \\
& (\nabla \cdot \boldsymbol{A})_{i}=(\operatorname{div} \boldsymbol{A})_{i}=A_{i j, j} \hat{e}_{i} \\
& (\nabla \times \boldsymbol{A})_{i j}=(\operatorname{curl} \boldsymbol{A})_{i j}=e_{j k l} A_{i l, k} \hat{e}_{i} \otimes \hat{e}_{j}
\end{aligned}
$$




\section{The dislocation transport IBVP}

Consider a simply-connected body $\mathcal{B}$ with boundary $\partial \mathcal{B}$ containing dislocations that are individually characterized by their Burgers vector $\boldsymbol{b}$ (Unit: $\mathrm{L}^{1}$ ) and the local dislocation line vector $\boldsymbol{l}\left(\mathrm{Unit}^{1}\right)$. The finite polar density $\boldsymbol{\alpha}:=\frac{1}{V} \boldsymbol{b} \otimes \boldsymbol{l}=\boldsymbol{b} \otimes \boldsymbol{t}$, where $V$ is a finite volume within which the polar dislocation density is non-zero and $\boldsymbol{t}=\boldsymbol{l} / V$ (Unit: $\mathrm{L}^{-2}$ ).

Let each dislocation move at a velocity $\boldsymbol{v}$. We are interested in their transport during a finite time span $t_{F}>0$ i.e., in the interval $\left[0, t_{F}\right]$. We restrict ourselves to an Eulerian description i.e., derivatives with respect to time are partial time-derivatives.

In the strong form, the dislocation transport IBVP is given as follows:

$$
\begin{array}{rlrl}
\dot{\boldsymbol{\alpha}}+\boldsymbol{\alpha} \cdot \boldsymbol{\mu}+(\nabla \boldsymbol{\alpha}) \cdot \boldsymbol{v}-(\nabla \cdot \boldsymbol{\alpha}) \otimes \boldsymbol{v}-\boldsymbol{S} & =\mathbf{0} & & \text { in } \mathcal{B} \times\left(0, t_{F}\right) \\
\nabla \cdot \boldsymbol{\alpha} & =\mathbf{0} & & \text { in } \mathcal{B} \times\left(0, t_{F}\right) \\
\boldsymbol{\alpha}(\cdot, t=0) & =\boldsymbol{\alpha}^{0} & \text { in } \mathcal{B} \\
\boldsymbol{\alpha} & =\boldsymbol{\gamma} & & \text { on } \partial \mathcal{B}^{-} \times\left(0, t_{F}\right) \\
\boldsymbol{\alpha} & =\mathbf{0} & & \text { on } \partial \mathcal{B}^{+} \times\left(0, t_{F}\right)
\end{array}
$$

Equation (17) is the dislocation transport equation, which is better known in the form [5, 6]: $\dot{\boldsymbol{\alpha}}+\nabla \times(\boldsymbol{\alpha} \times \boldsymbol{v})=\boldsymbol{S}$; equation (17) is obtained by expanding the curl of the cross-product between a tensor and a vector using the definitions in section 2. We prefer to use the dislocation transport equation as expressed in equation (1a) in order to develop the RKDG scheme and to understand the properties of the bilinear form.

$\boldsymbol{\mu}$ is a second order tensor such that $\boldsymbol{\mu}:=(\nabla \cdot \boldsymbol{v}) \mathbb{I}-(\nabla \boldsymbol{v})^{T}$, or in Einstein summation notation $\mu_{l j}:=\delta_{l j} v_{k, k}-v_{j, l}$, which gives $\boldsymbol{\alpha} \cdot \boldsymbol{\mu}=(\nabla \cdot \boldsymbol{v}) \boldsymbol{\alpha}-\boldsymbol{\alpha}$. $(\nabla \boldsymbol{v})^{T}$, or $\alpha_{i l} \mu_{l j}=\alpha_{i j} v_{k, k}-\alpha_{i k} v_{j, k} . \quad \boldsymbol{S}$ is the dislocation source term, which satisfies $\nabla \cdot \boldsymbol{S}=\mathbf{0}$.

Equation $(1 \mathrm{~b})$ is a continuity condition, which implies that a dislocation line can either end at $\partial \mathcal{B}$, be present in $\mathcal{B}$ in the form of a loop or branch in to/out of other dislocations. This continuity condition is respected at any instant in time, which can be deduced by noting that $\nabla \cdot \dot{\boldsymbol{\alpha}}=\mathbf{0}$.

The initial configuration of dislocations is given by equation $\left(1\right.$ ) where $\boldsymbol{\alpha}^{0}$ is the initial polar dislocation density field, which satisfies $\nabla \cdot \boldsymbol{\alpha}^{0}=\mathbf{0}$.

$\partial \mathcal{B}^{-}$and $\partial \mathcal{B}^{+}$are the inflow and outflow parts of $\partial \mathcal{B}$, respectively, such that

$$
\begin{aligned}
\partial \mathcal{B}^{-} & :=\{x \in \partial \mathcal{B} \mid \boldsymbol{v}(x) \cdot \hat{\boldsymbol{n}}(x)<0\} \\
\partial \mathcal{B}^{+} & :=\{x \in \partial \mathcal{B} \mid \boldsymbol{v}(x) \cdot \hat{\boldsymbol{n}}(x)>0\}
\end{aligned}
$$

where $\hat{\boldsymbol{n}}(x)$ is the normal to a point $x$ on a boundary.

On the inflow boundary $\partial \mathcal{B}^{-}$, we can prescribe a polar dislocation density $\gamma$ as done in equation $(1 \mathrm{~d})$; one can also prescribe a dislocation flux $\boldsymbol{\gamma}(\boldsymbol{v} \cdot \hat{\boldsymbol{n}})$, however, this term is simply a scaling of the dislocation density and for simplicity, but without the loss of generality, we use the condition $(1 \mathrm{~d})$. On the outflow boundary $\partial \mathcal{B}^{+}$, the condition (1) implies that dislocations should not leave the body; this condition is also a consequence of $\nabla \cdot \boldsymbol{\alpha}^{0}=\mathbf{0}$. 


\section{The RKDG approach for the dislocation transport IBVP}

The type of time-explicit RKDG scheme to be used to solve the dislocation transport IBVP (1) requires understanding the nature of equation (17), which in turn depends on the definition of $\boldsymbol{v}$. Based on a mechanical dissipation analysis performed by [6], an infinite number of expressions are permissible for the dislocation velocity as long as they satisfy $\int_{\mathcal{B}} \boldsymbol{f} \cdot \boldsymbol{v} \geq 0$ everywhere in the domain; $\boldsymbol{f}$ is the Peach-Koehler force that is related to $\boldsymbol{\alpha}$ and the stress tensor $\boldsymbol{\sigma}$ as $\boldsymbol{f}:=(\boldsymbol{\sigma} \cdot \boldsymbol{\alpha}): \mathrm{X}$. In this work, for simplicity and without the loss of generality, we assume that $\boldsymbol{v} \equiv \boldsymbol{v}(\boldsymbol{\alpha})$. This dependence of $\boldsymbol{v}$ on $\boldsymbol{\alpha}$ makes equation (17) a non-linear transport problem.

However, since we aim at obtaining a time-explicit RK scheme, we shall assume that $\boldsymbol{v}$ is dependent on the $\boldsymbol{\alpha}$ of the previous time step when we compute the $\boldsymbol{\alpha}$ of the current time step. Then, at any given time step, $\boldsymbol{v}$ can be considered as a known quantity in the evaluation of equation set (1) i.e., it can be considered as given data. Consequently, $\boldsymbol{\mu}$ can also be considered as given data. Then, equation (1a) has the form of a linear unsteady-state advection-reaction (no diffusion) hyperbolic partial differential equation with: (i) $(\nabla \boldsymbol{\alpha}) \cdot \boldsymbol{v}$ - the advection term, (ii) $\boldsymbol{\alpha} \cdot \boldsymbol{\mu}$ - the zeroth-order reaction term and, (iii) $(\nabla \cdot \boldsymbol{\alpha}) \otimes \boldsymbol{v}$ - a first-order term.

The method of lines approach [19, 20] is used to solve equation set (1). We have followed the procedure outlined in the work of Di Pietro and Ern [20] for hyperbolic partial differential equations, however, a step by step derivation is not presented here; interested readers may refer to [20] or other works.

\subsection{Variational formulation of the continuous problem}

The starting point of the method of lines approach in this work is the variational (weak) formulation of the continuous problem. In order to construct the continuous variational formulation of the dislocation transport IBVP, we need to first endow appropriate continuity and differentiability properties to all the variables entering equation set (1). These properties are crucial for determining the well-posedness of the problem [20].

We assume that $\boldsymbol{v}$ is a continuous and locally integrable function in space. We further assume that $\boldsymbol{\mu}$, which is a derivative of $\boldsymbol{v}$, exists in the weak sense and it is uniquely defined almost everywhere. These assumptions are particularly helpful when an expression of $\boldsymbol{v}$ allows it to be continuous but not differentiable (in the strong sense) everywhere; this case is encountered when using equation (20). Sobolev spaces gather the properties of locally integrable functions and weak derivatives.

Hence, formally, we assume [20] that $\boldsymbol{v} \in[\operatorname{Lip}(\Omega)]^{d}$ and $\boldsymbol{\mu} \in\left[L^{\infty}(\mathcal{B})\right]^{d \times d}$, where $d(=2$ or 3$)$ is the space dimension, $\operatorname{Lip}(\Omega)$ denotes the space spanned by Lipschitz continuous functions and $L^{\infty}(\mathcal{B})$ is a Banach space of essentially bounded measurable functions with the essential supremum norm. With this assumption for $\boldsymbol{v}$, there also holds $\boldsymbol{v} \in\left[W^{1, \infty}(\mathcal{B})\right]^{d}$ with the condition $\left\|\nabla v_{i}\right\|_{\left[L^{\infty}(\mathcal{B})\right]^{d}} \leq L_{v_{i}}, \forall i \in\{1, \ldots, d\}$; where $\left[W^{1, \infty}(\mathcal{B})\right]^{d}$ is a Sobolev vector space and $L_{v_{i}}$ is the Lipschitz module of component $v_{i}$. 
These assumptions will have consequences on the CFL condition tackled in section 5.2 .

Note that these assumptions do not take into account the time dependence of $\boldsymbol{v}$ and $\boldsymbol{\mu}$. Strictly speaking, $\boldsymbol{v}$ and $\boldsymbol{\mu}$ should be time-dependent, however, since they are assumed to be given data in the proposed time-explicit RKDG scheme, we make the concession of assuming that they are time-independent, which simplifies the derivation of the RKDG scheme [20].

Next, we assume that $\boldsymbol{S}$ is a continuous and at least once differentiable function in space, such that $\nabla \cdot \boldsymbol{S}$ exists so that $\nabla \cdot \boldsymbol{S}=\mathbf{0}$ can be satisfied. We also assume that $\boldsymbol{S}$ is continuous in time, however, it does not have to be differentiable in time. Formally, we assume $\boldsymbol{S} \in C^{0}\left(\left[H^{1}(\mathcal{B})\right]^{d \times d}\right)$, where $H^{1}(\mathcal{B})$ is the Hilbert space whose functions are continuous and at least once differentiable in space, and $C^{0}(\mathcal{V})$ is the space of $\mathcal{V}$-valued functions in space that are continuous in time but may or may not be differentiable.

$\gamma$ is assumed to be continuous in both space and time but not necessarily differentiable in either. Formally, $\gamma \in C^{0}\left(\left[L^{2}(|\boldsymbol{v} \cdot \hat{\boldsymbol{n}}| ; \partial \mathcal{B})\right]^{d \times d}\right)$, where $L^{2}(|\boldsymbol{v} \cdot \hat{\boldsymbol{n}}| ; \partial \mathcal{B}):=$ $\left\{w\right.$ is Lebesgue measurable $\left.\left|\int_{\partial \mathcal{B}}\right| \boldsymbol{v} \cdot \hat{\boldsymbol{n}} \mid w^{2}<\infty\right\}$.

The sought solution $\boldsymbol{\alpha}$ to the continuous variational problem is assumed to be continuous and at least once differentiable in both space and time. Formally, $\boldsymbol{\alpha} \in$ $C^{0}\left(\left[H^{1}(\mathcal{B})\right]^{d \times d}\right) \cap C^{1}\left(\left[L^{2}(\mathcal{B})\right]^{d \times d}\right)$, where $C^{1}(\mathcal{V})$ is the space of $\mathcal{V}$-valued functions in space that are continuous and at least once differentiable in time; following [20], it can be shown that if such a solution exists, then it is unique. Finally, it can be straightforwardly deduced that $\boldsymbol{\alpha}^{0} \in\left[H^{1}(\mathcal{B})\right]^{d \times d}$.

In brief, $\boldsymbol{\alpha}, \boldsymbol{S}$ and $\boldsymbol{\gamma}$ are functions of both space and time, and $\boldsymbol{v}, \boldsymbol{\mu}$ and $\boldsymbol{\alpha}^{0}$ are functions of only space. In the remainder of the article, the dependence on space and time of these functions will be implied and shall not be mentioned unless necessary.

Now, let $\boldsymbol{\eta} \in\left[H^{1}(\mathcal{B})\right]^{d \times d}$ be a time-independent test function. Defining $x^{\ominus}:=$ $\frac{1}{2}(|x|-x)$ as the negative part of a real number $x$, the continuous variational problem for equation set (1) is posed as:

For all $\boldsymbol{\eta} \in\left[H^{1}(\mathcal{B})\right]^{d \times d}$, find $\boldsymbol{\alpha} \in C^{0}\left(\left[H^{1}(\mathcal{B})\right]^{d \times d}\right) \cap C^{1}\left(\left[L^{2}(\mathcal{B})\right]^{d \times d}\right)$ such that

$$
\begin{aligned}
\int_{\mathcal{B}} \dot{\boldsymbol{\alpha}}: \boldsymbol{\eta} \mathrm{d} V & +a(\boldsymbol{\alpha}, \boldsymbol{\eta}) \\
& =\int_{\mathcal{B}} \boldsymbol{S}: \boldsymbol{\eta} \mathrm{d} V+\int_{\partial \mathcal{B}^{-}}(\boldsymbol{v} \cdot \hat{\boldsymbol{n}})^{\ominus} \boldsymbol{\gamma}: \boldsymbol{\eta} \mathrm{d} S, \forall t \in\left[0, t_{F}\right]
\end{aligned}
$$

with initial condition $\boldsymbol{\alpha}(\cdot, t=0)=\boldsymbol{\alpha}^{0}$. The continuous bilinear form $a(\boldsymbol{\alpha}, \boldsymbol{\eta})$ is defined as:

$$
\begin{aligned}
a(\boldsymbol{\alpha}, \boldsymbol{\eta}):= & \int_{\mathcal{B}}[\boldsymbol{\alpha} \cdot \boldsymbol{\mu}+(\nabla \boldsymbol{\alpha}) \cdot \boldsymbol{v}-(\nabla \cdot \boldsymbol{\alpha}) \otimes \boldsymbol{v}]: \boldsymbol{\eta} \mathrm{d} V \\
& +\int_{\partial \mathcal{B}}(\boldsymbol{v} \cdot \hat{\boldsymbol{n}})^{\ominus} \boldsymbol{\alpha}: \boldsymbol{\eta} \mathrm{d} S
\end{aligned}
$$

Following [20], it can be shown that the continuous variational problem (2) is 
well-posed i.e., it admits one and only one (unique) solution $\boldsymbol{\alpha} \in C^{0}\left(\left[H^{1}(\mathcal{B})\right]^{d \times d}\right) \cap$

$C^{1}\left(\left[L^{2}(\mathcal{B})\right]^{d \times d}\right)$. Furthermore, following proposition 2.7 in [20], it can be shown that when this $\boldsymbol{\alpha}$ solves (2), it solves (1) almost everywhere in $\mathcal{B}$ and $\partial \mathcal{B}$.

\subsection{Space semidiscretization: DG approach}

\subsubsection{Domain-level variational formulation}

In spirit of the method of lines approach, we first perform a space semidiscretization of the well-posed continuous variational problem (3). This procedure primarily involves discretizing the domain $\mathcal{B}$ using a mesh and choosing a local functional behavior in each mesh element; typically, polynomial functions are used, which is also the case in this work. Using the properties of the local functional behavior, the discrete bilinear form and the space semidiscrete variational problem can be obtained.

In what follows, the phrases shown in italic font are defined in Appendix A. We assume that the domain $\mathcal{B} \subset \mathbb{R}^{d}$ is a polyhedron in $\mathbb{R}^{d}$ and we discretize it using a general mesh $\mathcal{T}_{h}=\{T\}$ having a mesh size $h ; T \in \mathcal{T}_{h}$ is an element in $\mathcal{T}_{h}$ with boundary $\partial T$. Let $F$ be a mesh face, and $\mathcal{F}_{h}^{i}$ and $\mathcal{F}_{h}^{b}$ be the collection of all interfaces and boundary faces, respectively. Now, for an interface $F$ between two elements, let $\{\{\boldsymbol{\zeta}\}\}$ and $\llbracket \boldsymbol{\zeta} \rrbracket$ respectively represent the average and the jump of a second-order tensor $\boldsymbol{\zeta}$ for almost all $x \in F$; note that the average and jump operators act individually on each component of $\boldsymbol{\zeta}$. Let $n_{F}$ be the unit mesh normal to $F$ at almost all $x \in F$ for all $F \in \mathcal{F}_{h}$. Now, let $\mathbb{P}_{d}^{k}\left(\mathcal{T}_{h}\right)$ be the piecewise continuous vector space or broken polynomial space , here $k \geq 1$ is an integer that represents the polynomial degree. Finally, let $\mathcal{V}_{h}$ be the piecewise continuous test and solution space that is defined as $\mathcal{V}_{h}:=\left[\mathbb{P}_{d}^{k}\left(\mathcal{T}_{h}\right)\right]^{d}$.

We seek the approximate solution $\boldsymbol{\alpha}_{h} \in C^{0}\left(\mathcal{V}_{h}\right)$ to the space semidiscrete variational form that is presented below. Crucially, we continue to assume that $\boldsymbol{v}$ and $\boldsymbol{\mu}$ have the same functional dependencies as those presented in section 4.1; the reason is given at the end of this subsection. However, the space semidiscrete variational problem will use $\boldsymbol{S}_{h}:=\pi_{h} \boldsymbol{S}, \gamma_{h}:=\pi_{h} \gamma$ and $\boldsymbol{\alpha}_{h}^{0}:=\pi_{h} \boldsymbol{\alpha}^{0}$, where the operator $\pi_{h}$ projects a continuous field onto $\mathcal{V}_{h}$.

The space semidiscrete form of the continuous variational problem (2) is as follows:

For all test functions $\boldsymbol{\eta}_{h} \in \mathcal{V}_{h}$, find $\boldsymbol{\alpha}_{h} \in C^{0}\left(\mathcal{V}_{h}\right)$ such that

$$
\begin{aligned}
\int_{\mathcal{B}} \dot{\boldsymbol{\alpha}}_{h}: \boldsymbol{\eta}_{h} \mathrm{~d} V & +a_{h}^{\mathrm{upw}}\left(\boldsymbol{\alpha}_{h}, \boldsymbol{\eta}_{h}\right) d V \\
& =\int_{\mathcal{B}} \boldsymbol{S}_{h}: \boldsymbol{\eta}_{h} \mathrm{~d} V+\int_{\partial \mathcal{B}}(\boldsymbol{v} \cdot \hat{\boldsymbol{n}})^{\ominus} \boldsymbol{\gamma}_{h}: \boldsymbol{\eta}_{h} \mathrm{~d} S, \quad \forall t \in\left[0, t_{F}\right]
\end{aligned}
$$

with the initial condition $\boldsymbol{\alpha}_{h}(\cdot, t=0)=\boldsymbol{\alpha}_{h}^{0}$. $a_{h}^{\text {upw }}\left(\boldsymbol{\alpha}_{h}, \boldsymbol{\eta}_{h}\right)$ is the upwind DG bilinear form that is defined as: 


$$
\begin{aligned}
a_{h}^{\mathrm{upw}}\left(\boldsymbol{\alpha}_{h}, \boldsymbol{\eta}_{h}\right): & =\int_{\mathcal{B}}\left(\boldsymbol{\alpha}_{h} \cdot \boldsymbol{\mu}\right): \boldsymbol{\eta}_{h} \mathrm{~d} V \\
& +\int_{\mathcal{B}}\left[\left(\nabla_{h} \boldsymbol{\alpha}_{h}\right) \cdot \boldsymbol{v}-\left(\nabla_{h} \cdot \boldsymbol{\alpha}_{h}\right) \otimes \boldsymbol{v}\right]: \boldsymbol{\eta}_{h} \mathrm{~d} V \\
& +\int_{\partial \mathcal{B}}(\boldsymbol{v} \cdot \hat{\boldsymbol{n}})^{\ominus} \boldsymbol{\alpha}_{h}: \boldsymbol{\eta}_{h} \mathrm{~d} S \\
& -\sum_{F \in \mathcal{F}_{h}^{j}} \int_{F}\left(\boldsymbol{v} \cdot \hat{\boldsymbol{n}}_{F}\right) \llbracket \boldsymbol{\alpha}_{h} \rrbracket:\left\{\left\{\boldsymbol{\eta}_{h}\right\}\right\} \mathrm{d} S \\
& +\sum_{F \in \mathcal{F}_{h}^{i}} \int_{F} \frac{\kappa}{2}\left|\boldsymbol{v} \cdot \hat{\boldsymbol{n}}_{F}\right| \llbracket \boldsymbol{\alpha}_{h} \rrbracket: \llbracket \boldsymbol{\eta}_{h} \rrbracket \mathrm{d} S
\end{aligned}
$$

where $\kappa \geq 0$ is a user-specified penalty factor on the jumps in the trial and test functions across interfaces.

$\nabla_{h}$ is the broken gradient. Its usage implies that the derivatives exist and can be computed within each element of the mesh but not on the boundaries of the elements. According to its definition (see Appendix A.8), we have the second term on the right hand side of (5),

$$
\begin{aligned}
\int_{\mathcal{B}}\left[\left(\nabla_{h} \boldsymbol{\alpha}_{h}\right) \cdot \boldsymbol{v}-\left(\nabla_{h} \cdot \boldsymbol{\alpha}_{h}\right) \otimes \boldsymbol{v}\right]: \boldsymbol{\eta}_{h} \mathrm{~d} V \\
\quad \equiv \sum_{T \in \mathcal{T}_{h}} \int_{T}\left[\left(\nabla \boldsymbol{\alpha}_{h}\right) \cdot \boldsymbol{v}-\left(\nabla \cdot \boldsymbol{\alpha}_{h}\right) \otimes \boldsymbol{v}\right]: \boldsymbol{\eta}_{h} \mathrm{~d} V
\end{aligned}
$$

The upwind DG bilinear form (5) satisfies consistency, discrete coercivity and boundedness properties, and it delivers error estimates and (quasi-)optimal convergence rates for the smooth solution [20].

Note that the derivation of (5) exploits the continuity in space of the normal component of $\boldsymbol{v}$ across interfaces, which is one of the main reasons why we have assumed $\boldsymbol{v} \in\left[W^{1, \infty}(\mathcal{B})\right]^{d}$. If we were to assume that $\boldsymbol{v}$ belonged to the broken polynomial space

$\mathbb{P}_{d}^{k}\left(\mathcal{T}_{h}\right)$, then the bilinear form would not be the same as the one in (5) and its derivation would be more complicated.

Note also that the property $\nabla \cdot \dot{\boldsymbol{\alpha}}=\mathbf{0}$ is lost due to discretization because the solution can be discontinuous across mesh interfaces.

\subsubsection{Element-level variational formulation}

Some implementations of the DG problem involve recasting the domain-level space semidiscrete variational problem into an element-level one, which introduces the concept of numerical fluxes. While in this work we do not use an element-level implementation, it is nevertheless interesting to present this formulation in order to better understand some properties of the upwind DG bilinear form (5). 
For a real number $x$, let $x^{\oplus}:=\frac{1}{2}(|x|+x)$ define its "positive" part. Then, following the procedure outlined in [20], we obtain the following element-level space semidiscrete variational problem:

For all $\boldsymbol{\eta}_{h} \in \mathcal{V}_{h}$, find $\boldsymbol{\alpha}_{h} \in C^{0}\left(\mathcal{V}_{h}\right)$ such that

$$
\begin{aligned}
\int_{T} \dot{\boldsymbol{\alpha}}_{h}: \boldsymbol{\eta}_{h} \mathrm{~d} V+\sum_{F \in \mathcal{F}_{T}} \int_{F} \phi_{F}\left(\boldsymbol{\alpha}_{h}\right): \llbracket \boldsymbol{\eta}_{h} \rrbracket \mathrm{d} S \\
\quad-\int_{T}\left[\left(\boldsymbol{\alpha}_{h} \cdot(\nabla \boldsymbol{v})^{T}\right): \boldsymbol{\eta}_{h}+\left(\nabla \boldsymbol{\eta}_{h} \cdot \boldsymbol{v}\right): \boldsymbol{\alpha}_{h}+\left(\nabla \cdot \boldsymbol{\alpha}_{h}\right) \otimes \boldsymbol{v}\right] \mathrm{d} V \\
\quad=\int_{T} \boldsymbol{S}_{h}: \boldsymbol{\eta}_{h} \mathrm{~d} V, \quad \forall T \in \mathcal{T}_{h}, \forall t \in\left[0, t_{F}\right]
\end{aligned}
$$

where $\mathcal{F}_{T}$ is the collection of all faces belonging to the boundary $\partial T$ of $T$ (see definition in Appendix A.4, and $\phi_{F}$ are the numerical fluxes defined as:

$$
\phi_{F}\left(\boldsymbol{\alpha}_{h}\right):= \begin{cases}\left(\boldsymbol{v} \cdot \hat{\boldsymbol{n}}_{F}\right)\left\{\left\{\boldsymbol{\alpha}_{h}\right\}\right\}+\frac{\kappa}{2}\left|\boldsymbol{v} \cdot \hat{\boldsymbol{n}}_{F}\right| \llbracket \boldsymbol{\alpha}_{h} \rrbracket & \text { if } F \in \mathcal{F}_{h}^{i} \\ \left(\boldsymbol{v} \cdot \hat{\boldsymbol{n}}_{F}\right)^{\oplus} \boldsymbol{\alpha}_{h}-\left(\boldsymbol{v} \cdot \hat{\boldsymbol{n}}_{F}\right)^{\ominus} \boldsymbol{\gamma}_{h} & \text { if } F \in \mathcal{F}_{h}^{b}\end{cases}
$$

$\phi_{F}$ depends on the user-defined penalty parameter $\kappa \geq 0$. When $\kappa=0$, we get the centered fluxes $\boldsymbol{\phi}_{F}^{\text {cf }}$, which are so called because the average value of $\boldsymbol{\alpha}_{h}$ is used in computing them on each $F \in \mathcal{F}_{h}^{i}$. Usually, centered fluxes are not used because they result in a sub-optimal convergence rate. Choosing $\kappa=1$ leads to the so-called upwind fluxes in the context of finite volume schemes. It is recommended to not use a $\kappa \gg 1$; for a scalar space semidiscrete variational problem, Burman et al. [30] have shown that for matching simplicial meshes as $\kappa \rightarrow+\infty$, the discrete solution converges to the solution obtained from the CG approach, which is undesirable.

For any given fixed value of $\kappa$, the numerical fluxes $\phi_{F}$ are single-valued. Then, at the element-level, the space semidiscrete variational formulation is "conservative" in the sense that the $\boldsymbol{\alpha}_{h}$ that "flows" out of a mesh element through one of its faces "flows" into the adjacent element through that face. This property is one of the main reasons why the DG approach is an attractive one to solve transport-type problems.

\subsection{Time discretization: explicit $R K$ schemes}

The second and final step in the method of lines approach is to perform the time discretization of the space semidiscrete variational problem (4). The time discretization is performed using time-explicit RK schemes. Note that with a suitable basis for $\mathcal{V}_{h}$, problem (4) can be transformed into a system of coupled ordinary differential equations for the time-dependent components of $\boldsymbol{\alpha}_{h}$ on the chosen basis.

Let $\delta t$ be a constant time step such that $t_{F}=N \delta t$, where $N$ is the total number of time steps. For $n \in\{0,1, \ldots, N\}$ we define the discrete time $t^{n}=n \delta t$. A superscript $n$ to a function indicates its value at time $t^{n}$. 
Explicit RK schemes are often presented in $s$-stages with $p^{\text {th }}$ order of accuracy. They can be formulated in many different ways. Below, we will two popular approaches to formulate the $s$-stage RK scheme.

\subsubsection{Conventional approach}

Let $\boldsymbol{K}^{i}, i \in\{1, \ldots, s\}$, be second order tensors such that

$$
\begin{aligned}
\int_{\mathcal{B}} \boldsymbol{K}^{i}: \boldsymbol{\eta}_{h} \mathrm{~d} V= & -a_{h}^{\mathrm{upw}}\left(\boldsymbol{\alpha}_{h}^{n}+\delta t \sum_{j=1}^{s} a^{i j} \boldsymbol{K}^{j}, \boldsymbol{\eta}_{h}\right) \\
& +\int_{\mathcal{B}}\left(\boldsymbol{S}_{h}^{n+c^{i}}+\left(\boldsymbol{v}^{n} \cdot \hat{\boldsymbol{n}}\right)^{\ominus} \boldsymbol{\gamma}_{h}^{n+c^{i}}\right): \boldsymbol{\eta}_{h} \mathrm{~d} V \\
\boldsymbol{\alpha}_{h}^{n+1}= & \boldsymbol{\alpha}_{h}^{n}+\delta t \sum_{i=1}^{s} b^{i} \boldsymbol{K}^{i}
\end{aligned}
$$

where, $\boldsymbol{S}_{h}^{n+c^{i}}=\boldsymbol{S}_{h}\left(t^{n}+c^{i} \delta t\right)$ and $\boldsymbol{\gamma}_{h}^{n+c^{i}}=\boldsymbol{\gamma}_{h}\left(t^{n}+c^{i} \delta t\right) . a^{i j}$ is a matrix of real numbers, $b^{i}$ are real numbers such that $\sum_{i=1}^{s} b^{i}=1$, and $c^{i}$ are real numbers in the interval $[0,1]$ such that $c^{i}=\sum_{j=1}^{s} a^{i j}$, with $(1 \leq i, j \leq s)$.

The RK scheme is explicit whenever $a^{i j}=0$ for all $j \geq i$. Therefore, the summation on $j$ in equation set (8) is only up to $i-1$. The Butcher's tableau for the $s$-stage explicit RK scheme (8) is expressed as follows:

$$
\begin{array}{c|cccc}
c^{1} & 0 & 0 & \cdots & 0 \\
c^{2} & a^{21} & 0 & \cdots & 0 \\
\vdots & \vdots & \ddots & \ddots & \vdots \\
c^{s} & a^{s 1} & \cdots & a^{s, s-1} & 0 \\
\hline & b^{1} & \cdots & b^{s-1} & b^{s}
\end{array}
$$

\subsubsection{Strong Stability Preserving (SSP) approach}

An alternative approach, known as the SSP approach or the Total Variation Diminishing (TVD) approach, involves computing intermediate stages of the discrete solution instead of increments. Based on the work of Shu and Osher [21], the $s$-stage RK scheme for the dislocation transport IBVP without a source term or boundary conditions is given as:

$$
\begin{aligned}
\boldsymbol{\alpha}_{h}^{n, 0} & =\boldsymbol{\alpha}_{h}^{n} \\
\int_{\mathcal{B}} \boldsymbol{\alpha}^{n, i}: \boldsymbol{\eta}_{h} \mathrm{~d} V & =\sum_{j=0}^{i-1}\left(\int_{\mathcal{B}} d^{i j} \boldsymbol{\alpha}_{h}^{n, j}: \boldsymbol{\eta}_{h} \mathrm{~d} V-\delta t g^{i j} a_{h}^{\mathrm{upw}}\left(\boldsymbol{\alpha}_{h}^{n, j}, \boldsymbol{\eta}_{h}\right)\right) \\
\boldsymbol{\alpha}_{h}^{n+1} & =\boldsymbol{\alpha}_{h}^{n, s}
\end{aligned}
$$


where $\left(d^{i j}\right)_{1 \leq i \leq s, 0 \leq j \leq i-1}$ and $\left(g^{i j}\right)_{1 \leq i \leq s, 0 \leq j \leq i-1}$ are lower triangular matrices. The contributions of $\boldsymbol{S}_{h}$ and $\boldsymbol{\gamma}_{h}$ will be added later in section 5.1 for the different RK schemes that have been numerically implemented for the simulations performed in this work because these contributions depend on the order of the RK scheme used.

We are interested in RK schemes where $d^{i j}$ and $g^{i j}$ are non-negative and $d^{i j}$ is nonzero whenever $g^{i j}$ is non-zero. $d^{i j}$ has to fulfil the following consistency requirement:

$$
\sum_{j=0}^{i-1} d^{i j}=1, \quad \forall i \in\{1, \ldots, s\}
$$

As a consequence of these rules, the intermediate stages of the discrete solution, $\boldsymbol{\alpha}_{h}^{n, i}$, correspond to convex combinations of forward Euler substeps with the time step $\delta t$ replaced by $\frac{g^{i j}}{d^{i j}} \delta t$ resulting in the so-called SSP schemes. The SSP approach involves finding $d^{i j}$ and $g^{i j}$ such that $(9 \mathrm{~b})$ has the highest order of accuracy possible for that $s$-stage scheme. To that end, one approach is to use the conventional RK approach of (8) and rewrite it in the form of (9). However, most conventional RK-schemes result in small CFL numbers and negative $g^{i j}$ 's, which is undesirable [21, 31]. Another approach, proposed by Shu and Osher [21] and expanded by Gottleib and Shu [31], involves performing and analysing Taylor expansions of equation (9b) in order to find $d^{i j}$ and $g^{i j}$. Using this approach, Shu and Osher [21] developed SSP RKDG schemes of order 1, 2 and 3 with only positive $g^{i j}$. In this work, we use the $d^{i j}$ and $g^{i j}$ proposed by Shu and Osher [21]. 


\section{Numerical implementation}

In this section, we discuss the different $s$-stage RK schemes that have been numerically implemented in their SSP form (9) together with source and boundary terms, their CFL condition, slope limiting and the generic algorithm. The numerical implementation has been performed for the 3D case in the FEniCS [32, 33] open-source FE software using its Python interface.

\subsection{Explicit s-stage RK schemes}

\subsubsection{1-stage forward Euler scheme (RK1)}

The 1-stage RK scheme (RK1) is known as the forward Euler scheme. The coefficients $d^{i j}$ and $g^{i j}$ associated with this scheme are

$$
d=[1], \quad g=[1]
$$

which results in the following RK1-scheme

$$
\int_{\mathcal{B}} \boldsymbol{\alpha}^{n+1}: \boldsymbol{\eta}_{h} \mathrm{~d} V=\int_{\mathcal{B}} \boldsymbol{\alpha}^{n}: \boldsymbol{\eta}_{h} \mathrm{~d} V-\delta t a_{h}^{\mathrm{upw}}\left(\boldsymbol{\alpha}_{h}^{n}, \boldsymbol{\eta}_{h}\right)+\delta t \int_{\mathcal{B}} \boldsymbol{P}_{h}^{n}: \boldsymbol{\eta}_{h} \mathrm{~d} V,
$$

where $\boldsymbol{P}_{h}^{n}:=\left(\boldsymbol{S}_{h}^{n}+\left(\boldsymbol{v}^{n} \cdot \hat{\boldsymbol{n}}\right)^{\ominus} \boldsymbol{\gamma}_{h}^{n}\right)$.

Note here that (10) is obtained by adding the contribution of the source term and the boundary condition to (9). Note also that the forward Euler scheme obtained from (8) is the same as 10 .

\subsubsection{2-stage SSP RK scheme (RK2)}

The RK2 scheme has the following coefficients:

$$
d=\left[\begin{array}{cc}
1 & \\
1 / 2 & 1 / 2
\end{array}\right], \quad g=\left[\begin{array}{ll}
1 & \\
0 & 1 / 2
\end{array}\right]
$$

which gives the following generic RK2 scheme:

$$
\begin{aligned}
\int_{\mathcal{B}} \boldsymbol{\alpha}_{h}^{n, 1}: \boldsymbol{\eta}_{h} \mathrm{~d} V= & \int_{\mathcal{B}} \boldsymbol{\alpha}_{h}^{n}: \boldsymbol{\eta}_{h} \mathrm{~d} V-\delta t a_{h}^{\mathrm{upw}}\left(\boldsymbol{\alpha}_{h}^{n}, \boldsymbol{\eta}_{h}\right)+\delta t \int_{\mathcal{B}} \boldsymbol{P}_{h}^{n}: \boldsymbol{\eta}_{h} \mathrm{~d} V \\
\int_{\mathcal{B}} \boldsymbol{\alpha}_{h}^{n+1}: \boldsymbol{\eta}_{h} \mathrm{~d} V= & \frac{1}{2} \int_{\mathcal{B}}\left(\boldsymbol{\alpha}_{h}^{n}+\boldsymbol{\alpha}_{h}^{n, 1}\right): \boldsymbol{\eta}_{h} \mathrm{~d} V-\frac{1}{2} \delta t a_{h}^{\mathrm{upw}}\left(\boldsymbol{\alpha}_{h}^{n, 1}, \boldsymbol{\eta}_{h}\right) \\
& +\frac{1}{2} \delta t \int_{\mathcal{B}} \boldsymbol{\psi}_{h}: \boldsymbol{\eta}_{h} \mathrm{~d} V
\end{aligned}
$$

where $\boldsymbol{\psi}_{h} \equiv \boldsymbol{\psi}_{h}\left(\boldsymbol{S}_{h}, \boldsymbol{\gamma}_{h}\right)$ is such that it provides, for smooth $\boldsymbol{S}_{h}$ and $\boldsymbol{\gamma}_{h}$, a $2^{\text {nd }}$-order approximation of $\left(\boldsymbol{P}_{h}^{n}+\delta t \frac{\partial \boldsymbol{P}_{h}^{n}}{\partial t}\right) ; \boldsymbol{P}_{h}^{n}$ is defined just after equation 10 . 
Dislocation transport via a time-explicit RKDG FE scheme

The RK2 scheme (11) corresponds to the conventional 2-stage Heun's method when [20, 34]

$$
\boldsymbol{\psi}_{h}=\left(\boldsymbol{S}_{h}^{n+1}+\left(\boldsymbol{v}^{n} \cdot \hat{\boldsymbol{n}}\right)^{\ominus} \boldsymbol{\gamma}_{h}^{n+1}\right)
$$

\subsubsection{3-stage SSP RK scheme (RK3)}

The RK3 scheme [20, 34] has been implemented using:

$$
d=\left[\begin{array}{ccc}
1 & & \\
1 / 2 & 1 / 2 & \\
1 / 3 & 1 / 3 & 1 / 3
\end{array}\right], \quad g=\left[\begin{array}{ccc}
1 & & \\
0 & 1 / 2 & \\
0 & 0 & 1 / 3
\end{array}\right]
$$

which gives the following generic RK3 scheme:

$$
\begin{aligned}
\int_{\mathcal{B}} \boldsymbol{\alpha}_{h}^{n, 1}: \boldsymbol{\eta}_{h} \mathrm{~d} V= & \int_{\mathcal{B}} \boldsymbol{\alpha}_{h}^{n}: \boldsymbol{\eta}_{h} \mathrm{~d} V-\delta t a_{h}^{\mathrm{upw}}\left(\boldsymbol{\alpha}_{h}^{n}, \boldsymbol{\eta}_{h}\right)+\delta t \int_{\mathcal{B}} \boldsymbol{P}_{h}^{n}: \boldsymbol{\eta}_{h} \mathrm{~d} V \\
\int_{\mathcal{B}} \boldsymbol{\alpha}_{h}^{n, 2}: \boldsymbol{\eta}_{h} \mathrm{~d} V= & \frac{1}{2} \int_{\mathcal{B}}\left(\boldsymbol{\alpha}_{h}^{n}+\boldsymbol{\alpha}_{h}^{n, 1}\right): \boldsymbol{\eta}_{h} \mathrm{~d} V-\frac{1}{2} \delta t a_{h}^{\mathrm{upw}}\left(\boldsymbol{\alpha}_{h}^{n, 1}, \boldsymbol{\eta}_{h}\right) \\
& +\frac{1}{2} \delta t \int_{\mathcal{B}}\left(\boldsymbol{P}_{h}^{n}+\delta t \frac{\partial \boldsymbol{P}_{h}^{n}}{\partial t}\right): \boldsymbol{\eta}_{h} \mathrm{~d} V \\
\int_{\mathcal{B}} \boldsymbol{\alpha}_{h}^{n+1}: \boldsymbol{\eta}_{h} \mathrm{~d} V= & \frac{1}{3} \int_{\mathcal{B}}\left(\boldsymbol{\alpha}_{h}^{n}+\boldsymbol{\alpha}_{h}^{n, 1}+\boldsymbol{\alpha}_{h}^{n, 2}\right): \boldsymbol{\eta}_{h} \mathrm{~d} V-\frac{1}{3} \delta t a_{h}^{\mathrm{upw}}\left(\boldsymbol{\alpha}_{h}^{n, 2}, \boldsymbol{\eta}_{h}\right) \\
& +\frac{1}{3} \delta t \int_{\mathcal{B}} \boldsymbol{\psi}_{h}: \boldsymbol{\eta}_{h} \mathrm{~d} V
\end{aligned}
$$

In this case, $\boldsymbol{\psi}_{h} \equiv \boldsymbol{\psi}_{h}\left(\boldsymbol{S}_{h}, \boldsymbol{\gamma}_{h}\right)$ is such that it provides, for smooth $\boldsymbol{S}_{h}$ and $\boldsymbol{\gamma}_{h}$, a $3^{\text {rd }}$-order apporximation of $\left(\boldsymbol{P}_{h}^{n}+\delta t \frac{\partial \boldsymbol{P}_{h}^{n}}{\partial t}+\frac{1}{2} \delta t^{2} \frac{\partial^{2} \boldsymbol{P}_{h}^{n}}{\partial t^{2}}\right)$.

The RK3 scheme (12) corresponds to the conventional 3-stage Heun's method when [20, 34]:

$$
\begin{aligned}
\int_{\mathcal{B}} \boldsymbol{\psi}_{h}: \boldsymbol{\eta}_{h} \mathrm{~d} V= & \int_{\mathcal{B}}\left[-\frac{5}{4} \boldsymbol{P}_{h}^{n}+\frac{9}{4} \boldsymbol{P}_{h}^{n+{ }^{2} / 3}-\frac{1}{2} \delta t \frac{\partial \boldsymbol{P}_{h}^{n}}{\partial t}\right]: \boldsymbol{\eta}_{h} \mathrm{~d} V \\
& -\frac{3}{2} \delta t A_{h}^{\text {upw }}\left(\boldsymbol{P}_{h}^{n+1 / 3}-\boldsymbol{P}_{h}^{n}-\frac{1}{3} \delta t \frac{\partial \boldsymbol{P}_{h}^{n}}{\partial t}, \boldsymbol{\eta}_{h}\right),
\end{aligned}
$$

where $\boldsymbol{P}_{h}^{n+c^{i}}:=\left(\boldsymbol{S}_{h}^{n+c^{i}}+\left(\boldsymbol{v}^{n} \cdot \hat{\boldsymbol{n}}\right)^{\ominus} \boldsymbol{\gamma}_{h}^{n+c^{i}}\right)$ with $\boldsymbol{S}_{h}^{n+c^{i}}$ and $\boldsymbol{\gamma}_{h}^{n+c^{i}}$ defined just after equation (8) and $c^{i}=0,1 / 3$, or $2 / 3$.

\subsection{CFL conditions}

In order to understand the CFL conditions for the explicit RKDG scheme for the dislocation transport IBVP, it is important to first understand the time scale and mesh size involved in the problem. 
There are two inherent time scales associated with the dislocation transport problem. The first one is associated with the simulation time $t_{F}$, which is finite. The second one is associated with the steady-state part of the problem, which can be infinite.

Based on the assumptions made in section 4.1 for $\boldsymbol{v}$ and $\boldsymbol{\mu}$, we define the following time scale for the steady-state part of the problem:

$$
\tau_{c}:=\left[\max \left(\|\boldsymbol{\mu}\|_{\left[L^{\infty}(\mathcal{B})\right]^{d \times d}}, L_{v}\right)\right]^{-1}
$$

where

$$
L_{v}:=\max _{1 \leq i \leq d} L_{v_{i}}
$$

with $v_{i}$ being the components of velocity in the Cartesian basis of $\mathbb{R}^{d}$ and $L_{v_{i}}$ being the Lipschitz module of $v_{i}$. Since $\boldsymbol{\mu}$ and $L_{v}$ have the units of the reciprocal of time, $\tau_{c}$ represents the (fastest) time scale in the steady-state problem.

In the event that $\|\boldsymbol{\mu}\|_{\left[L^{\infty}(\mathcal{B})\right]^{d \times d}}=L_{v}=0$, which corresponds to the case of constant advective velocity without reaction, we get $\tau_{c}=\infty$. To avoid dealing with infinite time, we consider the following time scale [20]:

$$
\tau_{*}:=\min \left(t_{F}, \tau_{c}\right)
$$

which is always finite.

Next, we make the following (mild) assumption on the time step

$$
\delta t \leq \tau_{*}
$$

and note that the inequality $\delta t \leq t_{F}$ is trivially understood and the inequality $\delta t \leq \tau_{c}$ means the time step resolves the reference time.

Next, we define the following velocity magnitude:

$$
v_{c}:=\|\boldsymbol{v}\|_{\left[L^{\infty}(\mathcal{B})\right]^{d}}
$$

which corresponds to the fastest speed in the steady-state problem, and assume that we have quasi-uniform meshes with the smallest mesh size $h$ that can be interpreted as the diameter of a sphere (in $d=3$ or circle in $d=2$ ) inscribed in the smallest element of the mesh. We make the following mild assumption on $h$ :

$$
h \leq v_{c} \tau_{*} .
$$

The assumption $h \leq v_{c} \tau_{c}$ implies that the local Damköhler number is not too large (avoiding strong reaction regimes) and that the mesh size resolves the spatial variations of the advective velocity [20]. Meanwhile, the assumption $h \leq v_{c} t_{F}$ means that a dislocation advected at speed $v_{c}$ crosses at least one mesh element over the time interval $\left(0, t_{F}\right)$.

With the bounds (13) and (14), respectively, on $\delta t$ and $h$, we now define the usual or standard CFL condition [17, 18] on the time step as:

$$
\delta t \leq \rho \frac{h}{v_{c}}
$$


where $\rho$ is some positive real number.

Another useful CFL criterion is the so-called ${ }^{4} /{ }^{-}$CFL condition [20, 35]

$$
\delta t \leq \rho^{\prime} \tau_{*}^{-1 / 3}\left(\frac{h}{v_{c}}\right)^{4 / 3}
$$

where $\rho^{\prime}$ is some positive real number.

The value of $\rho$ (and $\rho^{\prime}$ ) depends on the type of RK scheme used. For the RK1 scheme, we assume that there exists a $\rho^{\mathrm{EUL}}$ independent of $\delta t, h, v_{c}, \boldsymbol{v}$ (consequently, $\boldsymbol{\mu}), \boldsymbol{S}$ and $\gamma$ such that $\rho \leq \rho^{\mathrm{EUL}}$. Under the usual CFL criterion (15), for $k=0$ (finite volume scheme), the dominant term in the error estimate for the RK1 scheme converges as $O\left(h^{1 / 2}\right)$ [20]. Consequently, for small $h$ i.e., $h \rightarrow 0$, the errors incurred when using the RK1 scheme become significant. In addition, the error estimate deteriorates in long time causing additional problems. Furthermore, for $k \geq 1$, the CFL criterion is more stringent, making this scheme impractical, as is evidenced later in section 6.1.1.

For the RK2 scheme with $k=1$, typically the usual CFL criterion (15) is used with a threshold $\rho^{\mathrm{RK} 2}$ whose properties are the same as those of $\rho^{\mathrm{EUL}}$. However, for $k \geq 2$, one should use the ${ }^{4} /{ }^{-}$CFL criterion [20, 35]. For all $k \geq 1$, the RK2 scheme enjoys better stability than the RK1 scheme.

For the RK3 scheme with $k \geq 1$, typically the usual CFL criterion (15) with a threshold $\rho^{\mathrm{RK} 3}$, whose properties are the same as those of $\rho^{\mathrm{EUL}}$, is sufficient.

\subsection{Slope limiting}

The upwinding SSP RKDG scheme (9) provides a stable solution for the dislocation transport IBVP problem whenever a piecewise-constant dislocation density field (i.e., polynomial degree $k=0$ ) is used. However, piecewise-constant functions are seldom used in practice due to the poor accuracy of the solution. Typically, higher-order spatial approximations of the solution i.e., $k \geq 1$, are used. Now, the upwinding RKDG scheme gives a stable solution for $k \geq 1$ provided smooth (gradually evolving in space) fields are used. In practice, however, space discretization induces some roughness in the solution. When rough solutions are sought with high-order space approximation $(k \geq 1)$, then spurious oscillations, known as Gibbs oscillations [22], are induced in the solution. These oscillations result in a deterioration of the solution over time irrespective of the RK scheme used. Slope limiters can be used to strongly diminish (or completely eliminate) these spurious oscillations [20].

For our simulations, we use the "hierarchical vertex-based" slope limiter, which has been originally developed by Kuzmin [23, 24] for scalar functions, and which has been implemented in the Oscellaris library by Landet [36, 37, 38] to be used with the FEniCS software. During a numerical simulation using any s-stage SSP RK scheme, at any given time step, the slope limiter (denoted using an operator $\Lambda$ ) is applied to the intermediate stages and the final solution. 
Note that our problem involves solving for $\boldsymbol{\alpha}_{h}$, a second-order tensor field, but the slope limiter implemented in Oscellaris has been designed for application to scalar fields. We overcome this limitation by imposing the slope limiter independently on each of the nine components of $\boldsymbol{\alpha}_{h}$. This approach is found to work well for the cases studied in this work; it becomes particularly relevant in the case of expansion of a polygonal loop in section 6.3 where two non-zero dislocation density tensor components depend on the evolution of each other. For general 3D simulations with dislocations on different slip systems, however, it may become necessary to design slope limiters for application on a second-order tensor field, which is beyond the scope of the present work.

\subsection{Algorithm}

The algorithm for a generic $s$-stage RKDG scheme to solve the dislocation transport IBVP is presented in Algorithm 1. We have assumed that a FE software already exists and it has the capability of defining CG as well as DG function spaces; e.g., FEniCS software [33], which is used in this work. We also assume that a slope limiter library is available for use in the FE software; e.g., the Oscellaris library [38] developed for FEniCS, which is used in this work. 
Algorithm 1: RKDG scheme for the dislocation transport IBVP

\section{Initialization:}

Mesh, time step, CG and DG function spaces (polynomial degree $k$ );

Define $\boldsymbol{v}$ as a function in CG vector space;

Define $\boldsymbol{\alpha}_{h}$ as a (trial) function in DG tensor space;

Define $\boldsymbol{\eta}_{h}$ as a (test) function in DG tensor space;

Define $a_{h}^{\text {upw }}\left(\boldsymbol{\alpha}_{h}, \boldsymbol{\eta}_{h}\right)$ as done in (5);

Define $\boldsymbol{S}$ and $\boldsymbol{\gamma}$ as functions in CG tensor space;

Define $\boldsymbol{S}_{h}$ as $\pi_{h} \boldsymbol{S}$ and $\boldsymbol{\gamma}_{h}$ as $\pi_{h} \boldsymbol{\gamma}$;

Define $\boldsymbol{\alpha}_{h}^{n+1}$ and $\boldsymbol{\alpha}_{h}^{n}$, current and previous time step densities, resepctively, as functions in DG tensor space;

Define $\boldsymbol{\alpha}_{h}^{0}$ as a function in DG tensor space;

\section{Main code:}

Set $t$ equal to 0 ;

Assign $\boldsymbol{\alpha}_{h}^{0}$ to $\boldsymbol{\alpha}_{h}^{n}$ to get $\Lambda \boldsymbol{\alpha}_{h}^{n}$;

while $t$ is less than or equal to $t_{F}$ do

Determine $\boldsymbol{v}^{n}$ using $\Lambda \boldsymbol{\alpha}_{h}^{n}$;

Increment $t$ by $\delta t$;

Assign $\Lambda \boldsymbol{\alpha}_{h}^{n}$ to $\Lambda \boldsymbol{\alpha}_{h}^{n, 0}$

Set $i$ equal to 0 ;

while $i$ is less than or equal to $s$-stages do

Solve (9b) with $\boldsymbol{S}_{h}$ and $\boldsymbol{\gamma}_{h}$ from section 5.1 to get $\boldsymbol{\alpha}_{h}^{n, i}$;

Apply slope limiter to $\boldsymbol{\alpha}_{h}^{n, i}$ to get $\Lambda \boldsymbol{\alpha}_{h}^{n, i}$;

Increment $i$ by 1 ;

end

Assign $\Lambda \boldsymbol{\alpha}_{h}^{n, s}$ to $\Lambda \boldsymbol{\alpha}_{h}^{n+1}$;

Write $\Lambda \boldsymbol{\alpha}_{h}^{n+1}$ to a file;

end

Result: $\Lambda \boldsymbol{\alpha}_{h}^{N}\left(\right.$ where $\left.N=t_{F} / \delta t\right)$ 


\section{Results and discussion}

To demonstrate the predictive capabilities of the 3D RKDG numerical implementation of the dislocation transport IBVP, we shall consider the following model cases: (i) transport of a single screw dislocation, (ii) annihilation of two oppositely signed screw dislocations and (iii) expansion of a polygonal dislocation loop.

\subsection{Transport of a screw dislocation: parametric study}

In this section, we perform a parametric study to obtain a qualitative understanding of the importance of the different tunable parameters of the RKDG scheme on the stability and accuracy (and hence, the error) of the numerical solution. The tunable parameters include the degree of space approximation (polynomial degree $k$ ), time approximation (RK1, RK2 and RK3), slope limiting $(\Lambda)$, upwinding penalty $(\kappa)$, space discretization (mesh size $h$ ) and time discretization (time step $\delta t$ ). For this study, we perform simulations for the simplest case i.e., the transport of a single screw dislocation. We also study the role of a constant uniform velocity field and a varying non-uniform velocity field.

Working with the Euclidean space $\mathbb{R}^{3}$ and a Cartesian coordinate system, we consider an infinitely-long single screw dislocation with line direction parallel to $\hat{e}_{3}$ such that $\boldsymbol{\alpha}=\alpha_{33} \hat{e}_{3} \otimes \hat{e}_{3}$. We assume that this dislocation is moving with a velocity along direction $\hat{e}_{1}$ such that $\boldsymbol{v}=v_{1} \hat{e}_{1}$. Then, assuming that $\boldsymbol{S}=\mathbf{0}$ and $\boldsymbol{\gamma}=\mathbf{0}$ on $\partial \mathcal{B}^{-}$, equation set (1) reduces to:

$$
\begin{aligned}
\dot{\alpha}_{33}+\alpha_{33,1} v_{1}+\alpha_{33} v_{1,1} & =0 \\
\alpha_{33,3} & =0 \\
\alpha_{33}(\cdot, t=0) & =\alpha_{33}^{0}
\end{aligned}
$$

Since $\alpha_{33,3}$ does not enter into equation $(17 \mathrm{a})$, therefore, if $\alpha_{33}^{0}$ satisfies $(17 \mathrm{~b})$, then equation $17 \mathrm{~b})$ is satisfied at any given time.

We assume that the initial dislocation density $\alpha_{33}^{0}$ is given by:

$$
\alpha_{33}^{0}(\boldsymbol{x}):= \begin{cases}1, & \text { if }\left|x_{1}-x_{c}\right| \leq r_{c} \text { and }\left|x_{2}-y_{c}\right| \leq r_{c} \\ 0, & \text { otherwise }\end{cases}
$$

where $r_{c}$ is the dislocation "core" size and $\boldsymbol{x}_{c} \equiv\left(x_{c}, y_{c}\right)$ is the location of the centroid of the dislocation density cylinder. Note that the above expression results in a rough initial dislocation density field with a square cross-section; the square shape is chosen to demonstrate that the RKDG scheme can also deal with sharp changes in solution. Note also that equation (18) satisfies condition $(17 \mathrm{~b})$ since there are no variations along $\hat{e}_{3}$; this implies that $\left.17 \mathrm{~b}\right)$ will be satisfied at any given time. Note however that in the simulations, we use $\boldsymbol{\alpha}_{h}^{0}:=\pi_{h} \boldsymbol{\alpha}^{0}$, which is not necessarily divergence free. 
Simulations are performed using the 3D numerical implementation of the RKDG model for the dislocation transport IBVP. However, the current implementation of the slope limiter (section 5.3) does not allow using the periodic boundary condition feature with the DG approach, which means that we cannot perform simulations for infinitelylong dislocations using the 3D code. A workaround to this problem is to significantly increase the domain size along the dislocation line direction and to use only one element for space discretization along that direction. To that end, we assume that the simulation box $\mathcal{B}$ has the dimensions $20 \mathrm{~nm} \times 4 \mathrm{~nm} \times 100000 \mathrm{~nm}$ and we discretize $\mathcal{B}$ using only one element along $\hat{e}_{3}$. Consequently, for this transport problem the divergence-free condition is respected even by the approximate solution. Simulations are performed using nonsymmetric (diagonally to the right) structured tetrahedral meshes. The parameters in expression (18) are: $r_{c}=0.5 \mathrm{~nm}, x_{c}=5 \mathrm{~nm}$ and $y_{c}=2 \mathrm{~nm}$. Therefore, $\alpha_{h 33}^{0}$ is in $\mathrm{nm}^{-1}$. The simulation time is $t_{F}=1$ sec.

Finally, two types of dislocation velocities are considered:

(i) Constant uniform (advective) velocity field $\boldsymbol{v}=v_{1} \hat{e}_{1}$ with $v_{1}$ (in $\mathrm{nm} / \mathrm{sec}$ )

$$
v_{1}(\boldsymbol{x}, t)=10, \quad \forall(\boldsymbol{x}, t) \in \mathcal{B} \times\left(0, t_{F}\right)
$$

For this case, we have $\tau_{c}=\infty$. Consequently, $\tau_{*}=t_{F}=1 \mathrm{sec}$ and $\delta t \leq 1 \mathrm{sec}$ must be respected together with the CFL condition (15) or (16) depending on $k$ and the RK scheme used. Furthermore, the term $\alpha_{33} v_{1,1}$ in equation $(17 \mathrm{a})$ becomes equal to 0 .

(ii) Time-varying non-uniform velocity field (in $\mathrm{nm} / \mathrm{sec}$ )

$$
v_{1}(\boldsymbol{x}, t)=10 \operatorname{sign}\left(\Lambda \alpha_{h 33}(\boldsymbol{x}, t)\right), \quad \forall(\boldsymbol{x}, t) \in \mathcal{B} \times\left(0, t_{F}\right),
$$

where the signum function (sign) is approximated as

$$
\operatorname{sign}(x) \approx \begin{cases}-1 & \text { if } x \leq-\varepsilon \\ x / \varepsilon & \text { if }-\varepsilon<x<\varepsilon \\ 1 & \text { if } x \geq \varepsilon\end{cases}
$$

where $0<\varepsilon<1$ is a user-defined number. We use a continuous approximation for the signum function because we have assumed that $\boldsymbol{v} \in\left[W^{1, \infty}(\mathcal{B})\right]^{d}$. This approximation allows $\boldsymbol{\mu}$ to exist in the weak sense and respect $\boldsymbol{\mu} \in\left[L^{\infty}(\mathcal{B})\right]^{d \times d}$. Then, we note that for equation (20), we get $\boldsymbol{\mu}=\mathbf{0}$. For all the simulations that use equation (20), we have chosen $\varepsilon=10^{-12} \mathrm{~nm}$. In this case, we have $\tau_{c}=0.1 \varepsilon$ sec, which leads to $\tau_{*}=\tau_{c}=10^{-13}$ sec and $\delta t \leq 10^{-13}$ sec should be respected. However, since this time step restriction is only imposed by those parts of the dislocation density field where $\left|\alpha_{h 33}\right|<10^{-12} \mathrm{~nm}^{-1}$, it is found that using a ( 7 or 8 orders of magnitude) higher time step that respects the CFL condition (15) or (16) gives satisfactory results (see sections 6.1.5 and 6.2).

Note that we have used $\Lambda \alpha_{h 33}$ in equation (20). This is done to circumvent the fluctuations in $\alpha_{h 33}$ about 0 caused by the Gibbs oscillations generated when $k \geq 1$ is used to approximate the rough portions of $\alpha_{h 33}$ i.e., close to the edges of the solution. 
Nevertheless, when expression (20) is used, we encounter an incompatibility in the function spaces on the left-hand side and the right-hand side because $\Lambda \boldsymbol{\alpha}_{h}$ belongs to the DG space whereas $\boldsymbol{v}$ is a function in the CG space. There are two approaches to overcome this problem: (i) compute $\operatorname{sign}\left(\Lambda \alpha_{h 33}(\boldsymbol{x}, t)\right)$ in DG space and then project it to the CG space to obtain $\boldsymbol{v}$, or (ii) first project $\Lambda \boldsymbol{\alpha}_{h}$ to the CG space and then insert this projection into (20) to get $\boldsymbol{v}$. We have tried both approaches and found that the second one is the most suitable. In the first approach, projecting $\left(\operatorname{sign} \Lambda \alpha_{h 33}(\boldsymbol{x}, t)\right)$ from DG to CG space results in some of the rough parts of the dislocation density field to have a velocity of the opposite sign from the rest simply because the projection of the sign function with $\varepsilon=10^{-12}$ results in some undesirable fluctuations. Consequently, parts of the same density field move in opposite directions, which is an incorrect solution. In the second approach, the sign function is applied on the projection of the slope limited solution. This approach works well due to the regularity of the slope limited solution (slope limiting introduces some smoothness to the rough parts of the solution); in this case, the impact of $\varepsilon=10^{-12}$ is unclear.

\subsubsection{Space and time approximation}

In this section, we focus on understanding the role of space approximation $(k)$ and time approximation (RK scheme) on the stability and accuracy of the solution. To that end, we consider the transport of a single screw dislocation with initial density given by (18) due to a constant uniform velocity field (19) in the domain. In this case, equation (17 a) reduces to $\dot{\alpha}_{33}+\alpha_{33,1} v_{1}=0$, whose solution is simply a translation of the screw dislocation line along $\hat{e}_{1}$ by a distance of $10 \mathrm{~nm}$.

To highlight the interplay between the space and time approximations, we perform simulations for $k=1$ and $k=2$ with the RK1, RK2 and RK3 schemes while fixing the remaining set of parameters: $\kappa=1$, no slope limiting, space discretization using $200 \times 40 \times 1$ elements with $h=0.05 \mathrm{~nm}$, and $\delta t=0.001 \mathrm{sec}$, which respects the usual CFL condition $\delta t \leq\left(\rho h / v_{1}=0.005\right)$ assuming $\rho=1$ for all the cases; the mesh associated with this space discretization can be visualized in Figure S1. Figures 1 and 2 show the contour and line plots, respectively, at $t=0.001 \mathrm{sec}$ and at $t=1 \mathrm{sec}$ for the different RK schemes with $k=1$ and $k=2$. In addition, the exact solution is plotted in Figure 2 .

For $k=1$ at $t=0.001$ sec i.e., after the first time step, RK1 has resulted in the transport of the dislocation density without any Gibbs oscillation. Meanwhile, for $k=2$, the RK1 scheme induces some Gibbs oscillations already at $t=0.001$ sec. In both cases, however, as the simulations progress, the RK1 predicted solution deteriorates into rapidly increasing spurious oscillations. Furthermore, the deterioration of the solution is faster for $k=2$ than for $k=1$.

Gibbs oscillations are also present in the solution predicted by RK2 and RK3 schemes throughout the simulation, however, the solutions do not deteriorate as in the RK1 case. Between RK2 and RK3, in general, the Gibbs oscillations are higher in 
amplitude for the former in comparison to the latter. The differences are smaller for $k=1$ but much larger for $k=2$.

Even though the solution of RK2 with $k=2$ does not deteriorate as much as the one from the RK1 scheme, it is nevertheless oscillatory. Furthermore, the oscillations are significantly more than those for $k=1$. The reason for this lies in the fact that while $\delta t=0.001$ sec may satisfy the usual CFL condition (15), it does not satisfy the more stringent $4 /{ }_{3}$-CFL condition (16). The latter condition imposes $\delta t \leq 0.00086 \rho^{\prime}$. If we were to generously assume that $\rho^{\prime}=1$, even then the $4 /{ }_{3}$-CFL condition is not satisfied. This example demonstrates why one should use the $4 /{ }_{3}$-CFL condition when using RK2 with $k=2$.

For a fixed $k$, improving the time approximation by using a higher-order RK scheme reduces the total error by reducing the error incurred from the time approximation, and improves the numerical solution as can be seen from Figure 1. Similarly, for a fixed RK scheme, increasing $k$ should reduce the total error by reducing the error incurred from the space approximation. This effect can be seen in Figure 2 by comparing the (nearly) vertical sections of the predicted and exact solutions for $k=1$ and 2 . However, it is overshadowed by the Gibbs oscillations becoming more pronounced; an increase in $k$ from 1 to 2 has increased the amplitude and number of cycles of the Gibbs oscillations, as seen in Figures 1 and 2 .

Despite these errors, the solutions predicted by the RK2 and the RK3 schemes result in a translation of the axis of the dislocation density field i.e., the dislocation line, to the right by $10 \mathrm{~nm}$, which is the expected solution. 


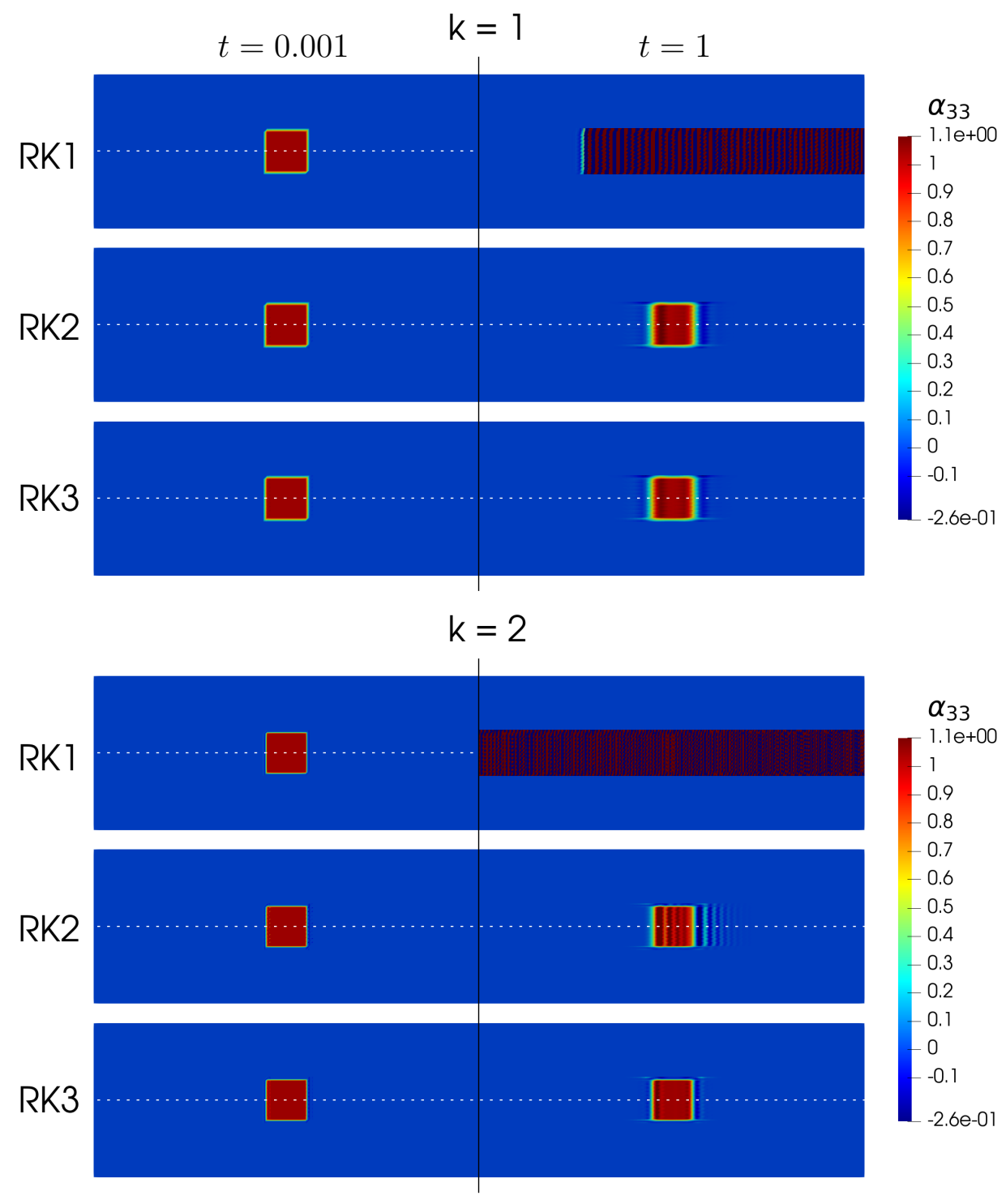

Figure 1: Role of space and time approximations: Comparison between predictions of RK1, RK2 and RK3 schemes for polynomial degree $k=1$ and $k=2$ (space approximations) for the transport of a single screw dislocation with density $\alpha_{33} \mathrm{~nm}^{-1}$ (initial state defined by (18)) at a constant velocity (19) without slope limiting. The parameters common to all these simulations are $\kappa=1$, no slope limiting, space discretization with $(200 \times 40 \times 1)$ elements, $t_{F}=1 \mathrm{sec}$ and $\delta t=0.001 \mathrm{sec}$. The black vertical lines denote the separation between the results after the first time step $(t=0.001 \mathrm{sec})$ and the last time step $(t=1 \mathrm{sec})$. The dashed lines are the lines along which the line plots in Figure 2 have been generated for the different RK schemes. This plot has been generated in ParaView 5.7.0. 

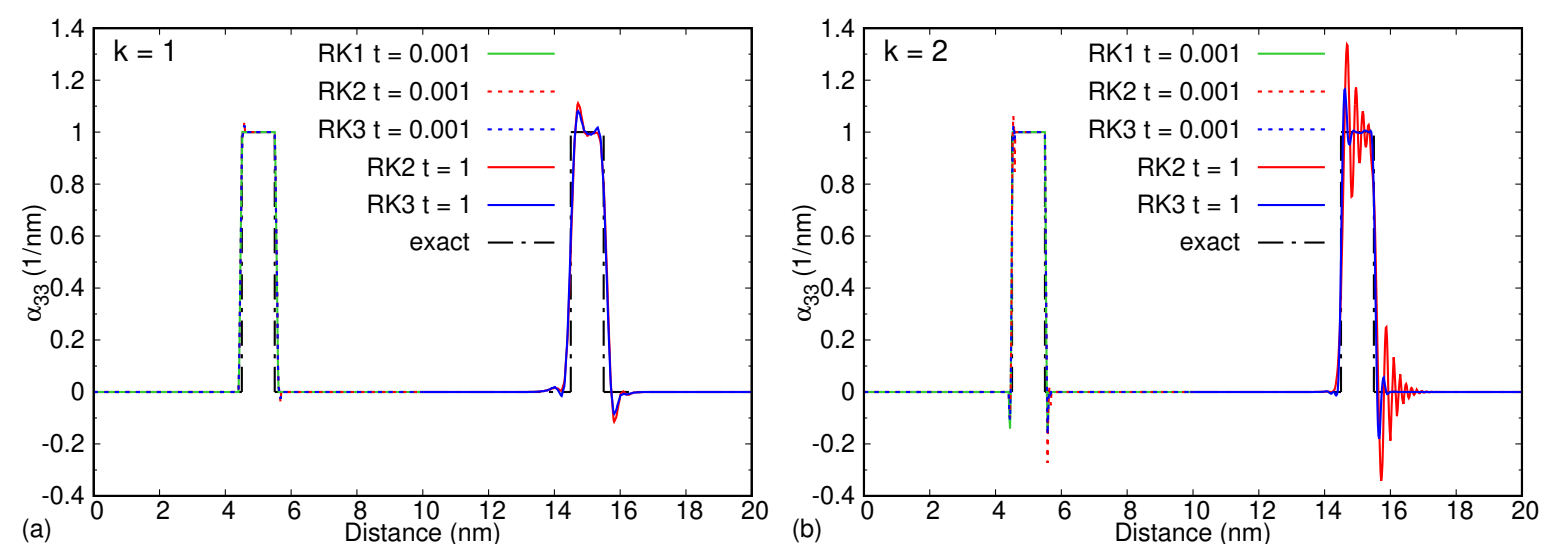

Figure 2: Line plot to understand role of space and time approximations: Line plot of $\alpha_{33}$ as a function of distance along the horizontal (dashed lines) lines bisecting the domains in Figure 1 for different RK schemes at $t=0.001 \mathrm{sec}$ and at $t=1 \mathrm{sec}$ with (a) $k=1$ and (b) $k=2$ in comparison with the exact solution. This plot has been generated using Gnuplot version 5.2.8.

\subsubsection{Slope limiter}

In order to diminish/eliminate the Gibbs oscillations encountered in the simulations performed in section 6.1.1, they are re-performed using the same parameters but this time with the slope limiter applied to the solution after each intermediate stage for every time step. Since the RK1 scheme resulted in complete deterioration of the solution, it does not make sense to use a slope limiter with this scheme that is naturally unstable. Therefore, only the results from RK2 and RK3 schemes are presented. The contour and line plots of the results of these simulations are shown in Figures 3 and 4 , respectively. The exact solution is plotted in Fig. 4 only along the shorter-spaced dashed lines (i.e., mid-section of the solution) in Figure 3 ; it is 0 at the so-called "top" portion of the solution.

Between $k=1$ and $k=2$, the final solution predicted using the latter is more diffuse and has a lower amplitude than the former, however, the final solution predicted using the former has slightly more fluctuations at the top portion of the dislocation density field. The larger spread and lower amplitude in the solution predicted by $k=2$ is a consequence of the corrections induced by the slope limiter to remove the stronger (higher amplitude and with more cycles) Gibbs oscillations than those in the solution with $k=1$. Continuing the discussion at the end of section 6.1.1, this result shows that after suppressing the Gibbs oscillations, increasing $k$ from 1 to 2 makes the solution slightly stabler but it also induces a slightly larger spread and a decrease in amplitude.

For both RK schemes, however, the bottom part of the final solution has vanished in the case when $k=2$ (Figure 3), which could be an artefact of the non-symmetric structured tetrahedral mesh used for the simulations.

In general, however, the solutions predicted by both RK2 and RK3 have a good match everywhere for both $k=1$ and $k=2$. This result implies that in this case 
not only does the slope limiter reduce the Gibbs oscillations but also it reduces the error contribution coming from the time approximation to the overall error. It also implies that with the slope limiter, the relative contribution from the error in space approximation to the overall error is significantly more than that coming from the time approximation. In order to reduce this imbalance, $k, \kappa$ and/or $\delta t / h$ should be increased; it was not possible to increase $k$ using the computing hardware available with us. The role of changing $\kappa$ is studied in section 6.1 .3 and that of $\delta t / h$ is studied in section 6.1.4.

An additional interesting consequence of using the slope limiter is that the ${ }^{4} /{ }$-CFL condition $(16)$ is no longer required for the simulations performed using the RK2 scheme with $k=2$ i.e., the usual CFL-condition is sufficient, at least in this specific case.



Figure 3: Role of slope limiter: Comparison between predictions of the RK2 and the RK3 schemes for polynomial degree $k=1$ and $k=2$ (space approximations) for the transport of a single screw dislocation with density $\alpha_{33} \mathrm{~nm}^{-1}$ (initial state defined by (18) ) at a constant velocity (19) with slope limiting. The other simulations parameters used are the same as those used to generate the results in Figure 1. The black vertical lines denote the separation between the results after the first time step $(t=0.001 \mathrm{sec})$ and the last time step $(t=1 \mathrm{sec})$. The dashed lines are the lines along which the line plots in Figure 4 have been generated. This plot has been generated in ParaView 5.7.0.

The results of this section show that applying the slope limiter strongly stabilizes the predicted solution for both $k=1$ and $k=2$. Henceforth, all the simulations will be 
performed using the slope limiter.
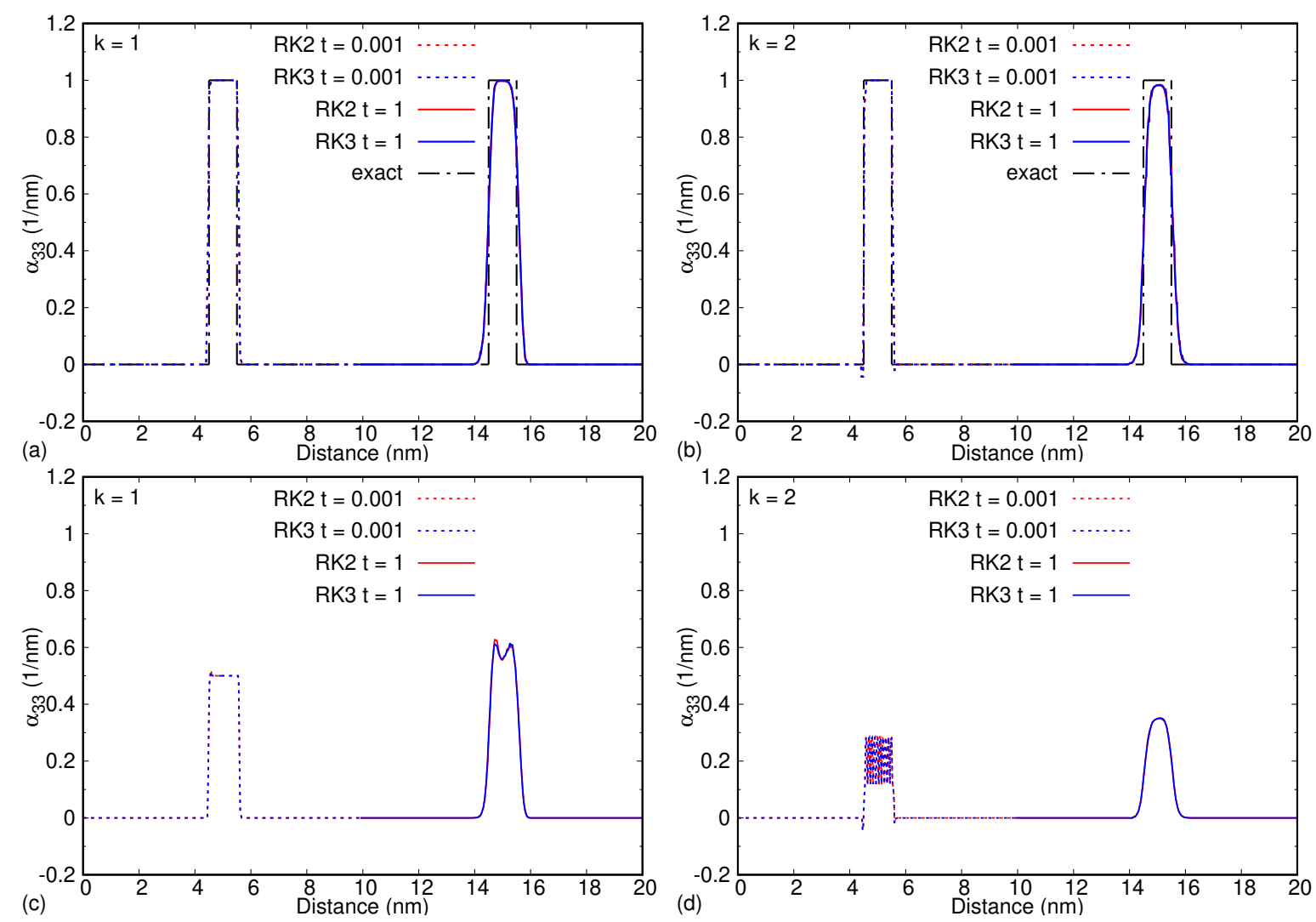

Figure 4: Line plots to understand role of slope limiter: Line plot of $\alpha_{33}$ as a function of distance along (a, b) shorter-spaced dashed lines and (c, d) wider-spaced dashed lines in Figure 3 for the RK2 and the RK3 schemes at $t=0.001$ sec and at $t=1$ sec with $(\mathrm{a}, \mathrm{c}) k=1$ and $(\mathrm{b}, \mathrm{d}) k=2$. The exact solution is shown only where it is relevant i.e., for $(a, b)$.

This plot has been generated using Gnuplot version 5.2.8.

\subsubsection{Upwinding penalty}

The upwinding penalty term in equation (5) strengthens the stability of the DG bilinear form to give quasi-optimal convergence rates. This term penalises the jumps in the trial and test solutions across internal facets with the help of a user adjustable parameter $\kappa$. In all the simulations performed so far, we have used $\kappa=1$, which corresponds to upwind fluxes in the context of finite volume schemes. As mentioned in section 4.2.2, when $\kappa=0$, we obtain the centered fluxes, and when $\kappa \rightarrow+\infty$, the DG predicted solution converges to the solution obtained from the CG approach. In general, it is recommended that $\kappa=0$ and $\kappa \gg 1$ should not be used. In order to find the upper limit of $\kappa$, the simulations performed with polynomial degree $k=1$ in section 6.1 .2 are re-performed with $\kappa=0,2$ and 3. Figure 5 shows the contour plot of the final state from each of these simulations. 


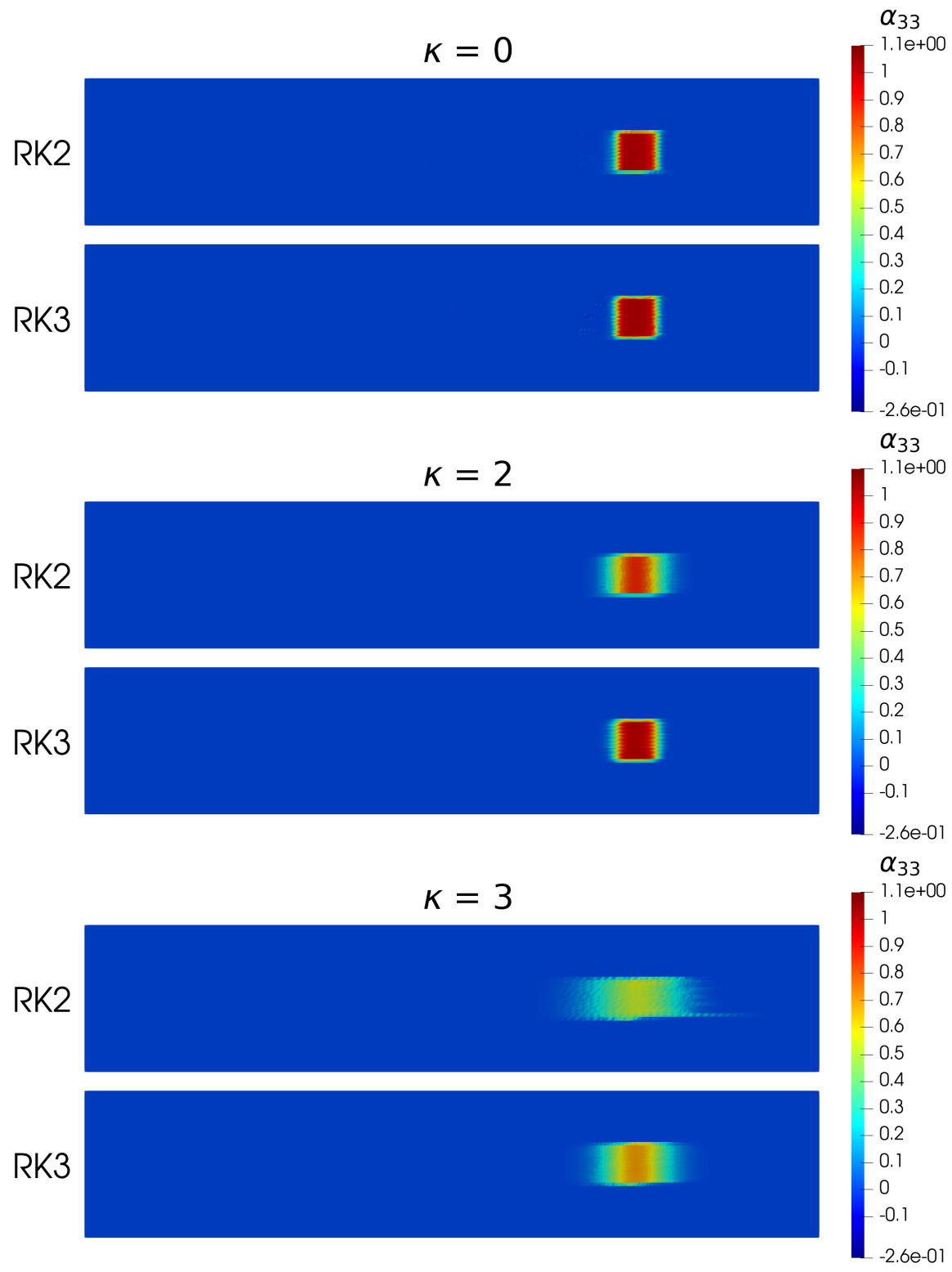

Figure 5: Role of upwinding penalty: Comparison between the final states predicted by the RK2 and the RK3 schemes for different upwinding penalties $\kappa=0,2,3$ for the transport of a single screw dislocation with density $\alpha_{33} \mathrm{~nm}^{-1}$ (initial state defined by (18) ) at a constant velocity (19) with slope limiting. The common parameters are $k=1$, slope limiting, space discretization with $(200 \times 40 \times 1)$ elements, $t_{F}=1$ sec and $\delta t=0.001$ sec. This plot has been generated in ParaView 5.7.0.

For all $\kappa$, the RK3 scheme results in a more accurate and less diffuse solution than RK2. For $\kappa=0$ i.e., centered fluxes, the solutions predicted by both schemes are less stable than the solutions predicted using $\kappa=1$ in Figure 3; although it is not visible in the Figure 5, parts of the dislocation density field trail far behind the rest, which is an incorrect solution. When $\kappa=2$ and $\kappa=3$ are used, both schemes transport the entire dislocation density. However, for $\kappa \geq 1$, the solution is increasingly unstable and diffuse 
with increasing $\kappa$. Based on these simulations, $\kappa=1$ i.e., upwind flux, appears to be the best choice. Note that these simulations were executed using the slope limiter, however, if a limiter were not to be used, then the solutions would have deteriorated significantly more and faster than with the limiter. In the limit case where $\kappa \rightarrow \infty$, one can deduce that a $\delta t \ll 0.001$ sec would be required to obtain a more compact solution even when using the RK3 scheme.

\subsubsection{Space and time discretization}

In this section, we perform two studies to understand (i) the role of space and time discretization on the predictive capabilities of RK2 and RK3 schemes while maintaining the same dimensionless constant $v_{c} \delta t / h$, and (ii) how close can $\delta t$ approach the upper bound set by the standard CFL condition before the solution completely deteriorates.

- From the CFL conditions, it is clear that if the number of elements were to be kept the same and the time step were to be decreased, then the solution predicted by any scheme would be more stable. Meanwhile, if the time step were to be kept the same and the number of elements were to be increased (at least along the direction of dislocation transport), then the solution predicted by all RK schemes would be less stable. Therefore, an approach to compare the role of space and time discretization on the predictive capabilities of the different RK schemes is to vary $\delta t$ and $h$ while keeping the dimensionless number $v_{c} \delta t / h$ constant and less than or equal to $\rho$ i.e., respect the usual CFL condition. In all the simulations performed so far, we have used $h=0.05 \mathrm{~nm}, \delta t=0.001 \mathrm{sec}$, and a constant uniform velocity field with $v_{c}=10 \mathrm{~nm} / \mathrm{sec}$ such that the dimensionless number $v_{c} \delta t / h=0.2$. We now discretize the domain into $100 \times 20 \times 1$ elements (see Figure S2) with $h=0.1$ $\mathrm{nm}$ and use $\delta t=0.002 \mathrm{sec}$ such that $v_{c} \delta t / h=0.2$ is respected. The remaining parameters are the same as those used in section 6.1.1. Figure 6 shows the results of these simulations.

Similar to the results for space discretization with $200 \times 40 \times 1$ elements and $\delta t=0.001 \mathrm{sec}$ in section 6.1.2, the RK2 and RK3 predicted solutions match very well, which indicates that the space approximation still has a higher error contribution than the time approximation.

While the solution is less rough (smaller spatial gradients) for the case of $100 \times 20 \times 1$ elements in comparison to the case of $200 \times 40 \times 1$ elements, the line plots (not shown) reveal that the spread in the dislocation density is larger for the former set of simulations for both RK schemes. It is indeed preferable to use a finer spatial resolution but doing that incurs a higher computational cost. Despite the larger spread, both schemes transport the entire dislocation density field such that the centroid has translated by $10 \mathrm{~nm}$. Therefore, if the purpose of the simulations is only to study the conservative property of the dislocation transport equation, then it suffices to use smaller number of elements with larger $\delta t$ in such a way that the dimensionless number $v_{c} \delta t / h \leq \rho$ remains constant. 




Figure 6: Role of space and time discretization keeping $v_{c} \delta t / h$ constant: Comparison between the final states predicted by the RK1, RK2 and RK3 schemes with $100 \times 20 \times 1$ elements $(h=0.1 \mathrm{~nm})$ and $\delta t=0.002 \mathrm{sec}$ for $k=1$ and $k=2$ for the transport of a single screw dislocation with density $\alpha_{33} \mathrm{~nm}^{-1}$ (initial state defined by (18)) at a constant velocity (19) with slope limiting. The parameters in common to the simulations performed for Figure 3 include slope limiting, $\kappa=1, t_{F}=1 \mathrm{sec}$ and the dimensionless number $v_{c} \delta t / h=0.2$. This plot has been generated in ParaView 5.7.0.

- In section 6.1.2, it was seen that the slope limiter increases the stability of the solutions predicted by both RK2 and RK3 in comparison to the case where no slope limiting is performed (section 6.1.1). In all cases, however, $\delta t$ respected the usual CFL condition. In this section, we study how much can $\delta t$ be increased before the slope limited solution becomes unstable. Similar to the previous case, we use a space discretization of $100 \times 20 \times 1$ elements with $h=0.1 \mathrm{~nm}$ and perform the simulations using (i) $\delta t=0.005 \mathrm{sec}$ such that $v_{c} \delta t / h=0.5$ and (ii) $\delta t=0.006667$ sec such that $v_{c} \delta t / h=0.6667$. We assume that the case of $\delta t=0.005 \mathrm{sec}$ is the limit case that satisfies the usual CFL condition $\delta t \leq 0.005 \rho$ with $\rho=1$ and the case of $\delta t=0.006667 \mathrm{sec}$ surpasses this limit. The remaining simulation parameters are $k=1, \kappa=1, t_{F}=1 \mathrm{sec}$ and with slope limiting. Therefore, $\delta t=0.005 \mathrm{sec}$ results in 200 time steps and $\delta t=0.006667$ sec results in 150 times steps. Figure 7 shows the results of these simulations. 
For both $\delta t=0.005 \mathrm{sec}$ and $\delta t=0.006667 \mathrm{sec}$, both RK2 and RK3 result in the transport of the dislocation density. However, there is a significantly larger spread in the solutions with $\delta t=0.006667 \mathrm{sec}$ than with $\delta t=0.005 \mathrm{sec}$. Furthermore, and in line with the discussion in section 6.1.2, we can now clearly see the advantage of using RK3 over RK2 in reducing the overall error in the solution.

When these simulations are re-performed without using the slope limiter (not shown), the solution predicted by both RK schemes deteriorates completely for both $\delta t=0.005 \mathrm{sec}$ and $\delta t=0.006667 \mathrm{sec}$. On one hand, these results demonstrate the stabilizing effect of the slope limiter. On the other hand, slope limiters must not be used on solutions that would otherwise deteriorate entirely. The time step to obtain a stable solution should be determined without slope limiting.

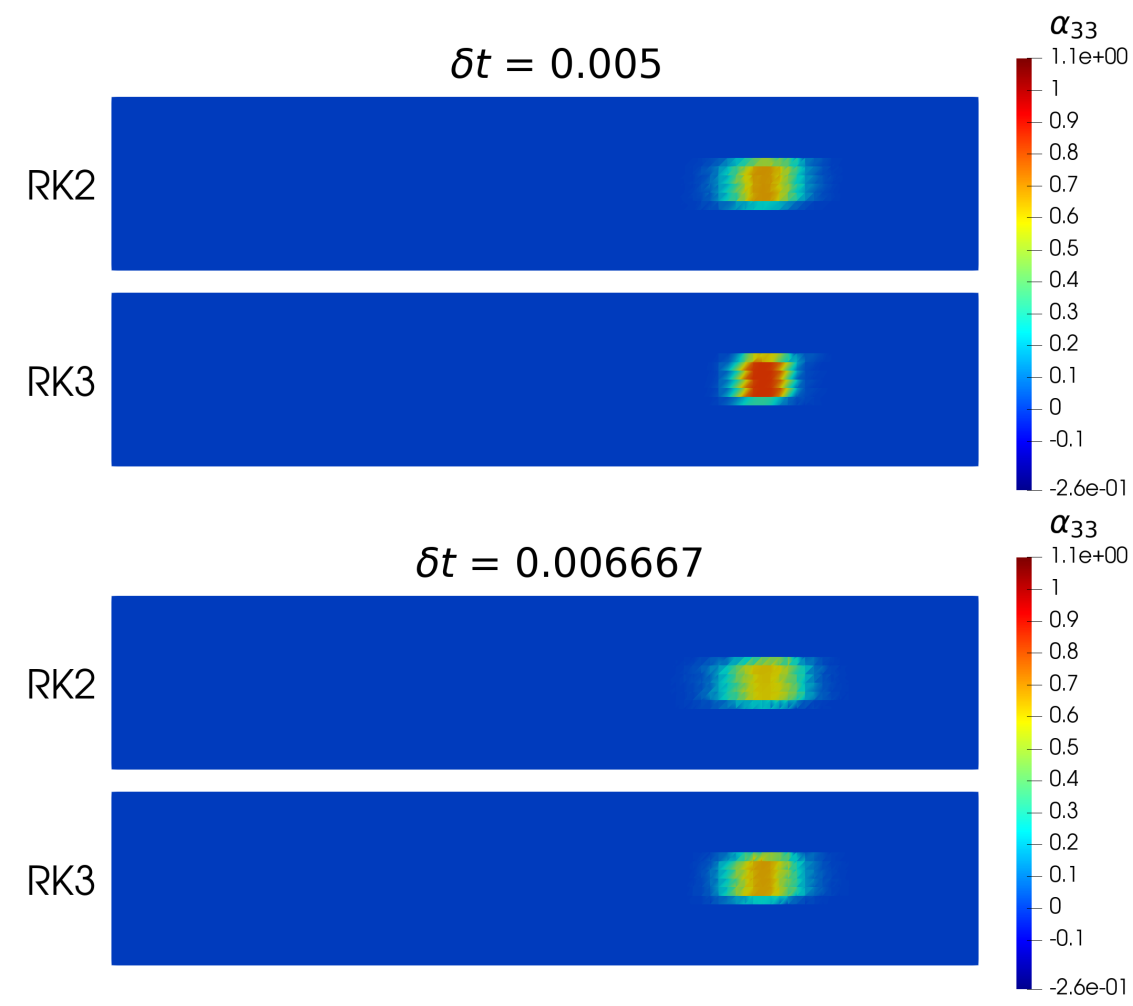

Figure 7: Effect of $\delta t$ approaching the CFL limit when using the slope limiter: Comparison between the final states predicted by the RK1, RK2 and RK3 schemes for $\delta t=0.005 \mathrm{sec}$ and $\delta t=0.006667 \mathrm{sec}$ for a space discretization of $100 \times 20 \times 1$ elements and the usual CFL condition (15) imposed upper limit being $0.005 \rho$ for the transport of a single screw dislocation with density $\alpha_{33} \mathrm{~nm}^{-1}$ (initial state defined by (18)) at a constant velocity (19). The remaining parameters common to these simulations are $k=1, \kappa=1$ and $t_{F}=1$ sec. This plot has been generated in ParaView 5.7.0.

\subsubsection{Non-uniform velocity field}

In this section, we re-perform the simulations performed in section 6.1 .2 , however, 
with a non-uniform varying velocity field given by equation 20.

In this case, the transport equation to be solved is the one given by equation (17a) and one of the two constraints on the time step is $\delta t \leq \tau_{*}=\min (0.1 h, 1)$. Since we are using $200 \times 40 \times 1$ elements, we have $h=0.05 \mathrm{~nm}$ and consequently, $\delta t$ has to be less than or equal to $\tau_{*}=0.005 \mathrm{sec}$, which is indeed the case since the simulations are performed using $\delta t=0.001$ sec.

The simulations are performed for both $k=1$ and $k=2$. Interestingly, $k=2$ results in a negligible difference with respect to the solutions predicted with constant velocity in Figure 3. Meanwhile, $k=1$ results in some noticeable differences. Figures 8 and 9 respectively show the contour and linear plots for the simulations with $k=1$.

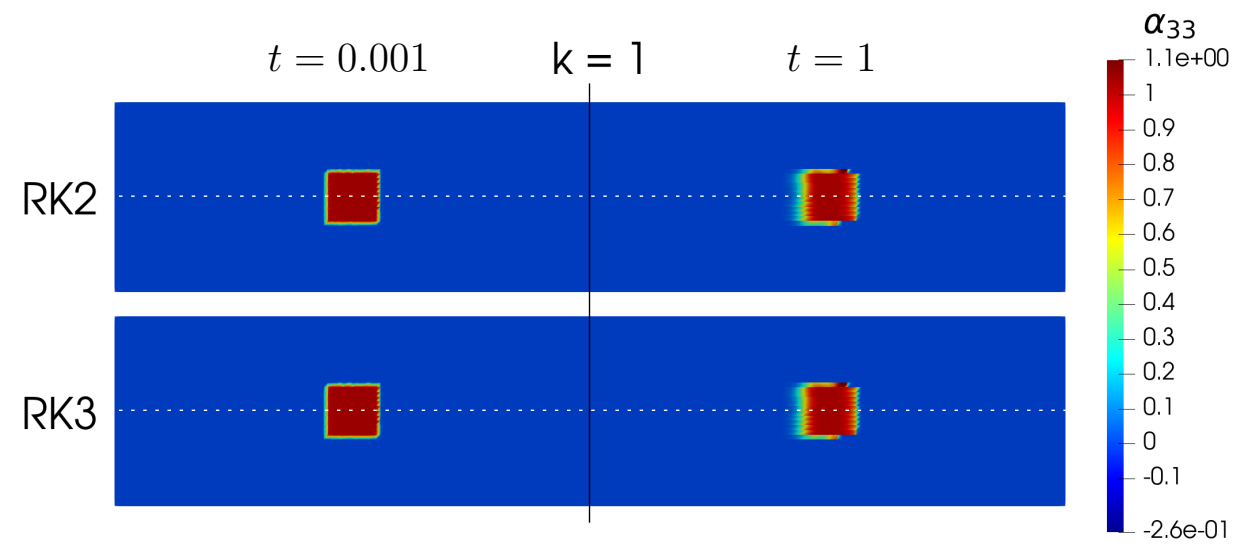

Figure 8: Role of non-uniform velocity: Comparison between predictions of the RK2 and RK3 schemes for polynomial degree $k=1$ for the transport of a single screw dislocation with density $\alpha_{33} \mathrm{~nm}^{-1}$ (initial state defined by (18)) with non-uniform velocity (20) and slope limiting. The parameters common to these simulations are $\kappa=1$, space discretization with $(200 \times 40 \times 1)$ elements, $t_{F}=1 \mathrm{sec}$ and $\delta t=0.001 \mathrm{sec}$. The black vertical lines denote the separation between the results after the first time step $(t=0.001 \mathrm{sec})$ and the last time step $(t=1 \mathrm{sec})$. The dashed lines are the lines along which the line plots in Figure 9 have been generated for the different RK schemes. This plot has been generated in ParaView 5.7.0.

The RK2 and RK3 predicted solutions match nearly perfectly everywhere. However, both schemes result in an overshoot at the top and bottom portions of the solution (Figure 8), which was not present in the solutions obtained from simulations performed with constant velocity. This difference should be a combination of the non-symmetry of the structured tetrahedral mesh and the term $\alpha_{33} v_{1,1}$, which was not present in the constant velocity case. Since $v_{1}$ is non-uniform and has large gradients at the extremities of the solution, the contribution of $\alpha_{33} v_{1,1}$ becomes significant at those locations. Comparing Figures 4(a) and 9, it can be seen that the solution with variable non-uniform velocity is slightly more asymmetric with respect to the exact solution than the one with the constant uniform velocity. Nevertheless, the dislocation density is transported by $10 \mathrm{~nm}$ in both simulations. 


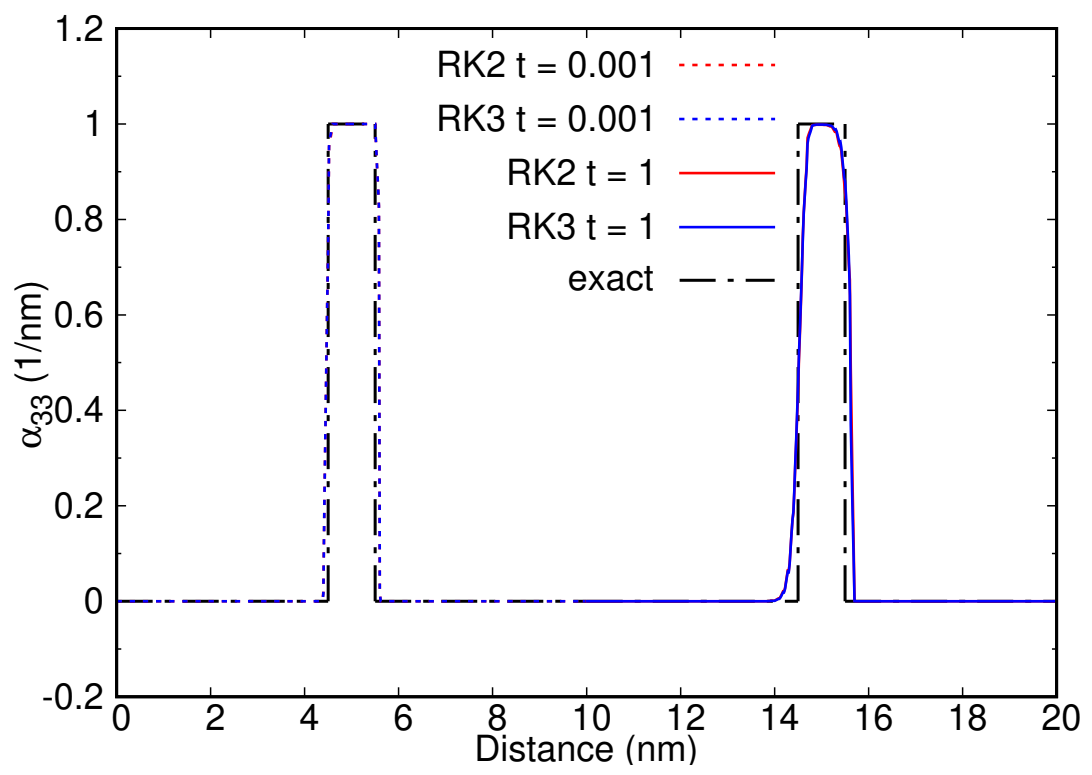

Figure 9: Line plot to study the role of non-uniform velocity: Line plot of $\alpha_{33}$ as a function of distance along dashed lines in Figure 8 for RK2 and RK3 schemes at $t=0.001 \mathrm{sec}$ and at $t=1 \mathrm{sec}$ with $k=1$ in comparison with the exact solution. This plot has been generated using Gnuplot version 5.2.8.

\subsection{Annihilation of two oppositely signed screw dislocations}

Consider two infinitely-long screw dislocations of opposite sign whose initial state is given by

$$
\alpha_{33}^{0}(\boldsymbol{x}):= \begin{cases}1, & \text { if }\left|x_{1}-x_{c}^{1}\right| \leq r_{c} \text { and }\left|x_{2}-y_{c}^{1}\right| \leq r_{c} \\ -1, & \text { if }\left|x_{1}-x_{c}^{2}\right| \leq r_{c} \text { and }\left|x_{2}-y_{c}^{2}\right| \leq r_{c} \\ 0, & \text { otherwise }\end{cases}
$$

where $\left(x_{c}^{1}, y_{c}^{1}\right)$ and $\left(x_{c}^{2}, y_{c}^{2}\right)$ are the location of the centroids of the dislocation density of the "positive" and "negative" screw dislocations, respectively. The simulation box dimensions and the number of elements are the same as those given at the beginning of section 6.1. $x_{c}^{1}=5 \mathrm{~nm}, y_{c}^{1}=2 \mathrm{~nm}, x_{c}^{2}=15 \mathrm{~nm}, y_{c}^{2}=2 \mathrm{~nm}$ and $r_{c}=0.5 \mathrm{~nm}$. Dislocation velocity is given by equation (20), which implies that the two dislocations are moving towards each other and will annihilate after translating $5 \mathrm{~nm}$ towards each other. Simulations are performed using the RK2 and RK3 schemes with at $\delta t=0.0001$ sec. The remaining RKDG parameters are the same as those used in section 6.1.2.

Figure 10 shows the snapshots of the solution predicted by RK3 at different time steps; the contour plot of the solution predicted by the RK2 scheme looks only slightly different from the one predicted by the RK3 scheme, and hence it is not shown; a video of the dislocation annihilation simulation using the RK3 approach is provided in the supplementary material. Figure 11 shows the lineplots of the solution at different time steps by both schemes. Both RK2 and RK3 schemes are able to resolve the shock generated from annihilation and both result in complete annihilation of dislocations at 
the end of the simulation; residual density of magnitude less than $10^{-4} \mathrm{~nm}^{-1}$ is left at the end of the simulation. However, the lineplots show that the RK2 simulation results in an overshoot in the solution due to the shock created at the location where the dislocation densities annihilate. Meanwhile, the solution predicted with RK3 has no such overshoot. In other words, the shock is resolved without any spurious oscillations by the RK3 scheme. This result highlights the importance of using a high-order RK scheme when dealing with discontinuities or shocks in the solution.



Figure 10: Dislocation annihilation: Comparison between the states at $t=0.001 \mathrm{sec}$, $t=0.5 \mathrm{sec}$ and $t=1 \mathrm{sec}$ predicted by the RK3 scheme for the dislocation annihilation simulation at $\delta t=0.0001 \mathrm{sec}$. The other parameters of this simulation are $k=1, \kappa=1$, slope limiting, space discretization with $(200 \times 40 \times 1)$ elements and $t_{F}=1 \mathrm{sec}$. The dashed lines are the lines along which the line plots in Figure 11 have been generated. This plot has been generated in ParaView 5.7.0.

\subsection{Expansion of a polygonal dislocation loop}

The polygonal loop lies in the same plane as its Burgers vector. Depending on the location on the loop, the dislocation can have either a screw, an edge or a mixed character; the character is determined by the angle between the local tangent to the dislocation line and the Burgers vector. This loop can either grow or shrink in the plane of its Burgers vector. In this section, we simulate the loop expansion.

In this problem, we consider an expanding dislocation loop with density $\boldsymbol{\alpha}=$ $\alpha_{11} \hat{e}_{1} \otimes \hat{e}_{1}+\alpha_{12} \hat{e}_{1} \otimes \hat{e}_{2}$ with the velocity $\boldsymbol{v}=v_{1} \hat{e}_{1}+v_{2} \hat{e}_{2}$. The component $\alpha_{11}$ is the screw component with the Burgers vector and line direction parallel to $\hat{e}_{1}$ and the component $\alpha_{12}$ is the edge component with the Burgers vector parallel to $\hat{e}_{1}$ and the 



Figure 11: Line plot to study dislocation annihilation: Line plot of $\alpha_{33}$ as a function of distance along dashed lines shown in Figure 10 from the solutions predicted by (a) the RK2 and (b) the RK3 schemes at $t=0.001 \mathrm{sec}, t=0.5 \mathrm{sec}$ and $t=1 \mathrm{sec}$. This plot has been generated using Gnuplot version 5.2.8.

line direction parallel to $\hat{e}_{2}$. Then, the equation set (1) becomes:

$$
\begin{aligned}
& \dot{\alpha}_{11}+\left(\alpha_{11} v_{2}-\alpha_{12} v_{1}\right)_{, 2}=0 \\
& \dot{\alpha}_{12}+\left(\alpha_{12} v_{1}-\alpha_{11} v_{2}\right)_{, 1}=0 \\
& \alpha_{11,1}+\alpha_{12,2}=0 \\
& \alpha_{11}(\cdot, t=0)=\alpha_{11}^{0} \\
& \alpha_{12}(\cdot, t=0)=\alpha_{12}^{0}
\end{aligned}
$$

The dislocation velocity components are assumed to be

$$
\begin{aligned}
v_{1} & =v_{0} \frac{\alpha_{12}}{\|\boldsymbol{\alpha}\|_{2}} \\
v_{2} & =-v_{0} \frac{\alpha_{11}}{\|\boldsymbol{\alpha}\|_{2}} \\
\text { with }\|\boldsymbol{\alpha}\|_{2} & =\sqrt{\alpha_{11}^{2}+\alpha_{12}^{2}}
\end{aligned}
$$

The dimensions of the domain are $40 \mathrm{~nm} \times 40 \mathrm{~nm} \times 0.8 \mathrm{~nm}$. The domain is discretized into $160 \times 160 \times 1$ elements using a symmetric structured tetrahedral mesh (see Figure S3). The dislocation loop is placed in the $x-y$ plane as shown in Fig. 12 . The center of the loop is at $(20 \mathrm{~nm}, 20 \mathrm{~nm})$ and the length of the inner part of the loop is $l=4 \mathrm{~nm}$. The thickness along the vertical and horizontal directions is $2 h=0.20366$ nm.

The initial state of the dislocation $\boldsymbol{\alpha}^{0}=\alpha_{11}^{0} \hat{e}_{1} \otimes \hat{e}_{1}+\alpha_{12}^{0} \hat{e}_{1} \otimes \hat{e}_{2}$ has to be such that equation $(22 \mathrm{k})$ is respected. To that end, we consider a dislocation loop having the configuration shown in Fig. 12. The top and bottom straight portions of the loop have (in $\mathrm{nm}^{-1}$ ) $\alpha_{11}^{0}=-1$ and $\alpha_{11}^{0}=1$, respectively, with $\alpha_{12}^{0}=0$. The left and right straight portions of the loop have $\alpha_{12}^{0}=-1$ and $\alpha_{12}^{0}=1$, respectively, with $\alpha_{11}^{0}=0$. The corners 
of the loops have:

$$
\begin{aligned}
& \alpha_{11}^{0}=-\frac{y-\left(y_{c}+b\right)}{\sqrt{\left(x-\left(x_{c}+a\right)\right)^{2}+\left(y-\left(y_{c}+b\right)\right)^{2}}} \\
& \alpha_{12}^{0}=\frac{x-\left(x_{c}+a\right)}{\sqrt{\left(x-\left(x_{c}+a\right)\right)^{2}+\left(y-\left(y_{c}+b\right)\right)^{2}}}
\end{aligned}
$$

where $\left(x_{c}, y_{c}\right)$ are the coordinates of the center of the loop and $(a, b)$ take the values of $(l, l),(-l, l),(-l,-l)$ and $(l,-l)$ in the top right, top left, bottom left and bottom right corners, respectively. The initial dislocation density is assumed to be uniform across the third direction.

This initial condition respects $\nabla \cdot \boldsymbol{\alpha}^{0}=\mathbf{0}$ everywhere in the continuous domain. However, unlike the simulations conducted in sections 6.1 and 6.2, this condition may not be respected at some points (notably the corners) in the discretized domain. Therefore, when performing the simulations for dislocation expansion, we have strongly imposed that $\nabla_{h} \cdot \boldsymbol{\alpha}=\mathbf{0}$ in equation (5). Note that unlike the cases studied in sections 6.1.5 and 6.2 , in the present case, $\boldsymbol{\mu} \neq \mathbf{0}$.

The simulation is performed using the RK2 scheme with $k=1, \kappa=1$ and slope limiting for a total time $t_{F}=5 \mathrm{sec}$ in 5000 steps such that $\delta t=0.0001$ sec. Since we are using $k=1$, the time step should respect the usual CFL condition (15): $\delta t \leq 0.010183 \rho^{R K 2}$. Assuming that $\rho^{R K 2}=0.1$, the chosen $\delta t$ is smaller by an order of magnitude than the upper limit imposed by the usual CFL condition. Hence, the scheme is expected to yield a stable solution, which it indeed does. The results of the simulation at different instances in time are shown in Fig. 12.

The results show that the predicted solution remains highly stable for the entire duration of the simulation. The initial dislocation density is spread across 5 cuboid blocks (see Figure S3) along the vertical and horizontal directions. At $t=1.45 \mathrm{sec}$ (not shown in Fig. 12), when the loop just touches the boundary of the domain, the density is spread across 7 cuboid blocks (see Figure S4) across the straight portions along the vertical and the horizontal directions. Furthermore, a line plot along the horizontal line bisecting the domain reveals that the peak amplitude of the dislocation density magnitude along the horizontal direction is $0.72 \mathrm{~nm}^{-1}$. At the end of the simulation, the dislocation density is completely annihilated at the free boundaries, which is the expected solution; a residue with a maximum magnitude less than $10^{-11} \mathrm{~nm}^{-1}$ is obtained at the end of the simulation; a video of this simulation is provided in the supplementary material.

Note that the very low diffusion of the solution and a highly stable loop expansion has been obtained in spite of the slope limiter being applied individually on $\alpha_{11}$ and $\alpha_{12}$ (see section 5.3 for explanation). Design and application of a slope limiter for second order tensors is expected to either give the same response or improve it. 
Dislocation transport via a time-explicit RKDG FE scheme



$t=0.5$

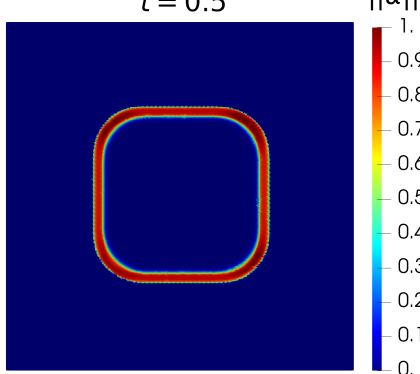

$t=1.0$
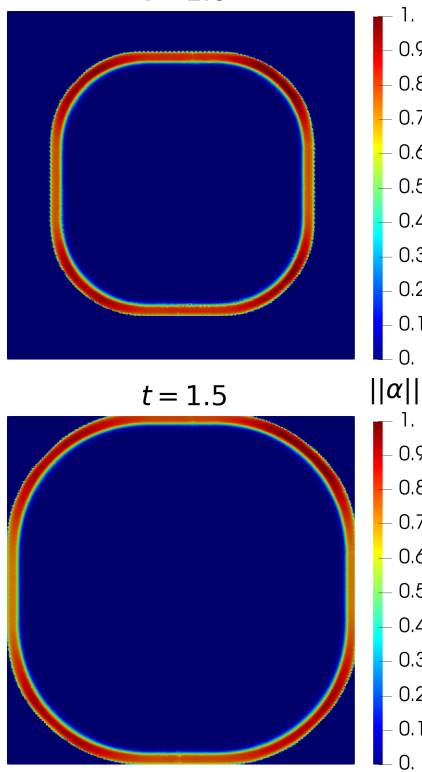

$t=2.0$



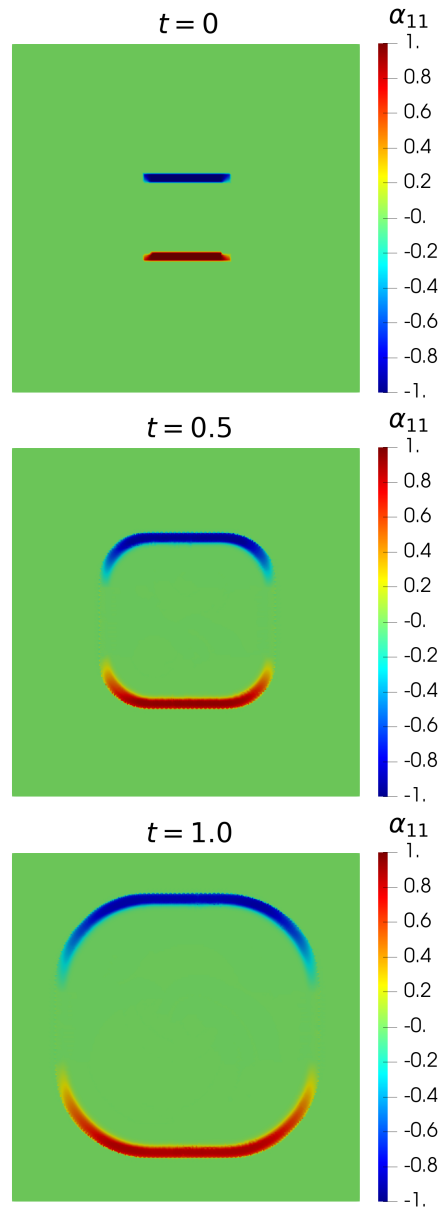

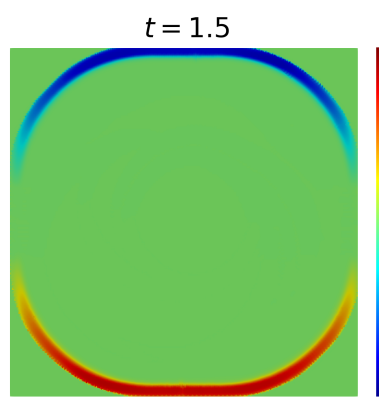

$t=2.0$

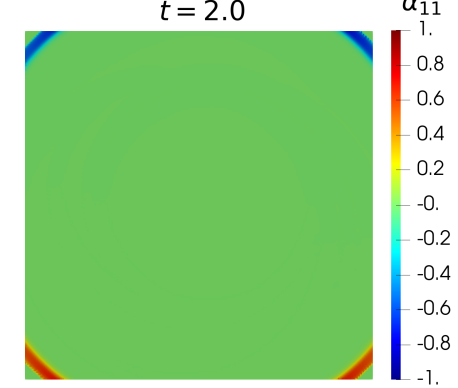

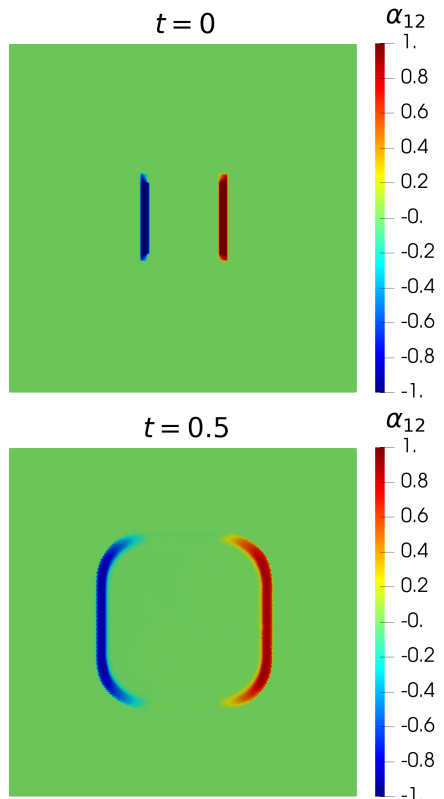
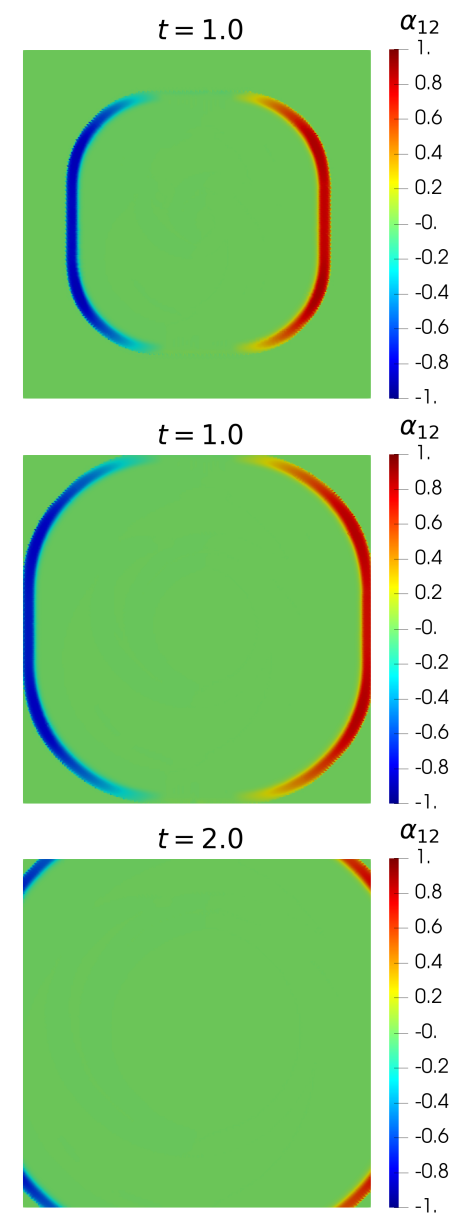

Figure 12: Expansion of a polygonal loop: Snapshots of the $L_{2}$-norm of $\boldsymbol{\alpha}\left(\|\boldsymbol{\alpha}\|_{2}\right)$, $\alpha_{11}$ and $\alpha_{12}$ at $t=0,0.5,1,1.5$ and 2 sec. This plot has been generated in ParaView 5.7.0. 


\section{Summary, conclusions and perspectives}

A time-explicit Runge-Kutta discontinuous Galerkin (RKDG) finite element (FE) approach is developed using the method of lines approach to solve the dislocation transport initial boundary value problem. Space semi-discretization is performed using the upwinding DG approach. Then, time discretization is performed using explicit RK schemes. The numerical implementation of the RKDG scheme is done for the 1stage first-order accurate RK (RK1 or forward Euler), 2-stage second-order accurate RK (RK2) and 3-stage third-order accurate RK (RK3) schemes using the Strong Stability Preserving (SSP) approach, also known as the Total Variation Diminishing (TVD) approach. The hierarchical vertex-based slope limiter is used to eliminate spurious Gibbs oscillations that always arise when rough solutions are approximated using highorder space approximations (polynomial degree $\geq 1$ ). The RKDG scheme is then applied to study the transport of a screw dislocation, annihilation of two oppositely signed screw dislocations and the expansion of a polygonal dislocation loop.

A parametric study is performed to understand the influence of key parameters of the RKDG scheme viz., space approximation (polynomial degree), time approximation (RK1, RK2 or RK3), slope limiter, upwinding penalty, space discretization (mesh size), time discretization (time step), and uniform constant or non-uniform variable velocity. In general, increasing the polynomial degree, improving the time approximation, and decreasing the time step and mesh size improved stability and accuracy of the solution. The solution predicted by the RK1 scheme rapidly deteriorates, underlining the importance of using higher-order accurate RK schemes for time discretization i.e., RK2 or RK3; due to its instability, the RK1 scheme was not used to perform other simulations. The slope limiter prevents the formation and propagation of spurious oscillations from the very first time step, thus stabilizing the RK2 and RK3 predicted solutions. The upwinding penalty factor of 1 was found to yield the most stable solution for the transport problem. Both uniform constant velocity and non-uniform variable velocity result in the expected dislocation transport.

With appropriate choice of simulations parameters, the proposed RKDG scheme is able to stably solve the dislocation annihilation problem. Specifically, it is able to resolve the shock generated during dislocation annihilation without inducing any spurious oscillations in the solution. At the end of the annihilation process, the residual dislocation density is significantly lower (4 orders of magnitude) than the magnitude of the initial density.

The simulated expansion of the polygonal dislocation loop is highly stable and with very low diffusion of the solution across elements. At the end of the simulation, the dislocation loop is annihilated at the free boundaries of the simulation domain, which

is the expected result. Furthermore, a negligibly small amount of residual is obtained (11 orders of magnitude lower than the magnitude of the initial density).

All of these results show the accuracy and robustness of the explicit RKDG FE approach in solving the dislocation transport IBVP. It allows accurately and robustly 
treating the short-range interactions between dislocations, which from a numerical standpoint has been a difficult problem to solve using polar dislocation density based approaches. One of the next steps will be to compare the simulation predictions and computational time of the RKDG approach with those from the existing 3D implementations of the dislocation transport IBVP via the continuous Galerkin/LeastSquares (CG-LS) [10, 11] and the Fast Fourier Transform (FFT) [14] numerical techniques, in order to understand the accuracy, the robustness and the domain of applicability of each approach. Another step could be to check how much error is being made by strongly imposing the continuity condition in the dislocation transport equation and whether it is important to correct it, for example, by incorporating locally divergence-free conditions into the solution space, as done in the work of Cockburn et al. [39]. Yet another step could be to extend the current (quasi-)linear formulation to the non-linear case where the dislocation velocity is a function of the dislocation density of the current time step. There are many challenges associated with this extension, mainly arising from the physical interpretation of the non-linear flux term across interfaces in the mesh, that one would need to address prior to its implementation; another factor to be considered is whether this extension could provide a significant improvement over the existing approach by taking into account the robustness of the (quasi-)linear scheme proposed in this work. Eventually, the RKDG approach will be implemented to solve the dislocation mechanics problem in the context of the isothermal field dislocation mechanics model [6] and then the thermal field dislocation mechanics model [7].

\section{Acknowledgements}

Manas V. Upadhyay is grateful to the European Research Council (ERC) for their support through the European Union's Horizon 2020 research and innovation programme for project GAMMA (Grant agreement No. 946959). The authors would like to express their profound gratitude to Prof. Alexandre Ern (who co-authored [20]) for the many stimulating discussions on the RKDG approach during the course of this work. 


\section{Appendix A. Definitions}

The definitions given below are adopted from the work of Di Pietro and Ern [20]. They are recalled here, often times verbatimly, in order to make the article standalone.

\section{Appendix A.1. Polyhedron}

A set $P$ is a polyhedron in $\mathbb{R}^{d}$ if it is an open, connected, bounded subset of $\mathbb{R}^{d}$ such that its boundary $\partial P$ is a finite union of parts of hyperplanes, say $\left\{H_{i}\right\}_{1 \leq i \leq n_{P}}$. A hyperplane is a subspace whose dimension is one less than that of the space under consideration; e.g., if a space is $d$-dimensional then its hyperplanes are the $(d-1)$-dimensional planes. Furthermore, for all $1 \leq i \leq n_{P}$, at each point in the interior of $\partial P \cap H_{i}$, the set $P$ is assumed to lie on only one side of its boundary.

\section{Appendix A.2. General mesh}

A general mesh $\mathcal{T}$ of the domain $\mathcal{B}$ is a finite collection of disjoint polyhedra $\{T\}$, such that $\mathcal{T}=\{T\}$, forming a partition of $\mathcal{B}$,

$$
\overline{\mathcal{B}}=\cup_{T \in \mathcal{T}} T
$$

Each $T \in \mathcal{T}$ is a mesh element. This definition of $\mathcal{T}$ includes non-degenerate (simplicial) meshes i.e., simplicial meshes are a subset of a general mesh. While the continuous Galerkin method is well suited for simplicial meshes, the DG method is well suited for any general mesh.

\section{Appendix A.3. Element diameter or meshsize}

For all $T \in \mathcal{T}, h_{T}$ denotes the diameter of the sphere (circle for $d=2$ ) inscribed in $T$, and the meshsize is defined as the real number

$$
h:=\max _{T \in \mathcal{T}} h_{T}
$$

The notation $\mathcal{T}_{h}$ is used for a mesh $\mathcal{T}$ with meshsize $h$.

Appendix A.4. Mesh faces

A closed subset $F$ of $\overline{\mathcal{B}}$ is a mesh face if $F$ has positive $(d-1)$-dimensional Hausdorff measure (in dimension 1 , this means that $F$ is nonempty) and if either one of the two following conditions is satisfied:

(i) $F$ is called an interface if there are distinct mesh elements $T_{1}$ and $T_{2}$ such that $F=\partial T_{1} \cap \partial T_{2}$.

(ii) $F$ is called a boundary face if there is a $T \in \mathcal{T}_{h}$ such that $F=\partial T_{1} \cap \partial \mathcal{B}$. 
Interfaces are collected in the set $\mathcal{F}_{h}^{i}$, and boundary faces are collected in the set $\mathcal{F}_{h}^{b}$ such that $\mathcal{F}_{h}^{i} \cap \mathcal{F}_{h}^{b}$ is a null set. Furthermore, the set

$$
\mathcal{F}_{T}:=\left\{F \in \mathcal{F}_{h} \mid F \subset \partial T\right\}
$$

collects the mesh faces composing the boundary of $T$.

Appendix A.5. Interface averages and jumps

We now define averages and jumps across interfaces of piecewise smooth functions. Let $\zeta_{i j}$ be a second-order tensor-valued function defined on $\mathcal{B}$ and assume that $\zeta_{i j}$ is smooth enough on all $F \in \mathcal{F}_{h}^{i}$, a possibly two-valued face, which means that $\forall T \in \mathcal{T}_{h}$, the restriction $\left.\zeta_{i j}\right|_{T}$ of $\zeta_{i j}$ to the open set $T$ can be defined up to the boundary $\partial T$. Then, $\forall F \in \mathcal{F}_{h}^{i}$ and for almost all $\boldsymbol{x} \in F$, the average of $\zeta_{i j}$ is defined as:

$$
\left\{\left\{\zeta_{i j}\right\}_{F}(\boldsymbol{x}):=\frac{1}{2}\left(\left.\zeta_{i j}\right|_{T_{1}}(\boldsymbol{x})+\left.\zeta_{i j}\right|_{T_{2}}(\boldsymbol{x})\right)\right.
$$

and the jump of $\alpha_{i j}$ as

$$
\llbracket \zeta_{i j} \rrbracket_{F}(\boldsymbol{x}):=\left.\zeta_{i j}\right|_{T_{1}}(\boldsymbol{x})-\left.\zeta_{i j}\right|_{T_{2}}(\boldsymbol{x})
$$

Note that the subscript $F$ has been dropped in the main text of this work; i.e., we simply write $\left\{\left\{\zeta_{i j}\right\}\right\}$ and $\llbracket \zeta_{i j} \rrbracket$.

Appendix A.6. Face normals

For all $F \in \mathcal{F}_{h}^{i} \cup \mathcal{F}_{h}^{b}$ and almost all $\boldsymbol{x} \in F$, we define the (unit) normal to $F$ at $\boldsymbol{x}$ as

(i) $\hat{\boldsymbol{n}}_{F}$, the unit normal to $F$ at $\boldsymbol{x}$ pointing from $T_{1}$ to $T_{2}$ if $F \in \mathcal{F}_{h}^{i}$ with $F=\partial T_{1} \cap \partial T_{2}$; the orientation of $n_{F}$ is arbitrarily depending on the choice of $T_{1}$ and $T_{2}$, but kept fixed once that choice has been made. Note that $n_{F}$ will always point towards the element with the higher index.

(ii) $\hat{\boldsymbol{n}}$, the unit outward normal to $\mathcal{B}$ at $\boldsymbol{x}$ if $F \in \mathcal{F}_{h}^{b}$.

Appendix A.7. The broken polynomial space

Let $x^{a}$ be such that for $\boldsymbol{x}=\left(x_{1}, x_{2}, \ldots, x_{d}\right) \in \mathbb{R}^{d}, x^{a}:=\prod_{i=1}^{d} x_{i}^{a_{i}}$, where $\boldsymbol{a}=\left(a_{1}, a_{2}, \ldots, a_{d}\right) \in$ $\mathbb{N}^{d}$. Then, we define the space of polynomials of $d$ variables, of total degree at most $k$, as

$$
\begin{aligned}
& \mathbb{P}_{d}^{k}(\mathcal{T}):= \\
& \quad\left\{p: \mathbb{R} \ni \boldsymbol{x} \mapsto p(\boldsymbol{x}) \in \mathbb{R} \mid \exists\left(\gamma_{\boldsymbol{a}}\right)_{\boldsymbol{a} \in A_{d}^{k}} \in \mathbb{R}^{\operatorname{card}\left(A_{d}^{k}\right)} \text { s.t. } p(\boldsymbol{x})=\sum_{\boldsymbol{a} \in A_{d}^{k}} \gamma_{\boldsymbol{a}} x^{a}\right\},
\end{aligned}
$$

where $A_{d}^{k}:=\left\{\left.\boldsymbol{a} \in \mathbb{N}^{d}|| \boldsymbol{a}\right|_{l^{1}} \leq k\right\}$ with $|\boldsymbol{a}|_{l^{1}}:=\sum_{i=1}^{d}\left|a_{i}\right|$. 
The dimension of the vector-space $\mathbb{P}_{d}^{k}$ is

$$
\operatorname{dim}\left(\mathbb{P}_{d}^{k}\right)=\operatorname{card}\left(A_{d}^{k}\right)={ }^{k+d} C_{k}=\left(\begin{array}{c}
k+d \\
k
\end{array}\right)=\frac{(k+d) !}{k ! d !}
$$

For example, the dimension of $\mathbb{P}_{3}^{2}$ is 10 because there are 10 possible combinations of the $x, y$ and $z$ dimensions, namely, $1, x, y, z, x^{2}, y^{2}, z^{2}, x y, y z$, and $x z$.

The broken polynomial space is then defined as

$$
\mathbb{P}_{d}^{k}\left(\mathcal{T}_{h}\right):=\left\{\boldsymbol{x} \in\left[L^{2}(\mathcal{B})\right]^{d}\left|\forall T \in \mathcal{T}_{h}, \boldsymbol{x}\right|_{T} \in \mathbb{P}_{d}^{k}(\mathcal{T})\right\}
$$

$\mathbb{P}_{d}^{k}(T)$ is spanned by the restriction to $T$ of polynomials in $\mathbb{P}_{d}^{k}$. The dimension of $\mathbb{P}_{d}^{k}\left(\mathcal{T}_{h}\right)$ is

$$
\operatorname{dim}\left(\mathbb{P}_{d}^{k}\left(\mathcal{T}_{h}\right)\right)=\operatorname{card}\left(\mathcal{T}_{h}\right) \times \operatorname{dim}\left(\mathbb{P}_{d}^{k}\right)
$$

Appendix A.8. The broken gradient

The definition of the broken gradient requires defining the broken Sobolev space

$$
\mathbb{W}^{k, p}\left(\mathcal{T}_{h}\right):=\left\{w \in L^{p}(\mathcal{B})\left|\forall T \in \mathcal{T}_{h}, w\right|_{T} \in W^{k, p}(T)\right\}
$$

where $W^{k, p}(T)$ is a Sobolev space.

We define the broken gradient $\nabla_{h}: W^{1, p}\left(\mathcal{T}_{h}\right) \mapsto\left[L^{p}(\mathcal{B})\right]^{d}$ such that for all $w \in W^{1, p}\left(\mathcal{T}_{h}\right)$,

$$
\forall T \in \mathcal{T}_{h},\left.\quad\left(\nabla_{h} w\right)\right|_{T}:=\nabla\left(\left.w\right|_{T}\right)
$$

The subscript $h$ in $\nabla_{h}$ is dropped whenever this operator appears inside an integral over a fixed mesh element $T \in \mathcal{T}_{h}$. 


\section{References}

[1] Hull D and Bacon D 2011 Introduction to dislocations 5th ed (Butterworth-Heinemann)

[2] Anderson P M, Hirth J P and Lothe J 2017 Theory of Dislocations (Cambridge University Press)

[3] Nye J F 1953 Acta Metallurgica 1 153-162

[4] Mura T 1963 Philosophical Magazine 8 843-857

[5] Acharya A 2001 Journal of the Mechanics and Physics of Solids 49 761-784

[6] Acharya A 2003 Proceedings of the Royal Society of London. Series A: Mathematical, Physical and Engineering Sciences 459 1343-1363

[7] Upadhyay M V 2020 Journal of the Mechanics and Physics of Solids 145104150

[8] Johnson C 2009 Numerical solution of partial differential equations by the finite element method (New York, NY, USA: Dover Publications, Inc.)

[9] Gottlieb D and Hesthaven J S 2001 Journal of Computational and Applied Mathematics 128 $83-131$

[10] Roy A and Acharya A 2005 Journal of the Mechanics and Physics of Solids 53 143-170

[11] Varadhan S N, Beaudoin A J, Acharya A and Fressengeas C 2006 Modelling and Simulation in Materials Science and Engineering 14 1245-1270

[12] Arora R, Zhang X and Acharya A 2020 Computer Methods in Applied Mechanics and Engineering 367113076

[13] Hughes T J R, Franca L P and Hulbert G M 1989 Computer Methods in Applied Mechanics and Engineering 73 173-189

[14] Djaka K S, Taupin V, Berbenni S and Fressengeas C 2015 Modelling and Simulation in Materials Science and Engineering 23065008

[15] Das A, Acharya A and Suquet P 2016 Computational Mechanics 57 387-403

[16] Morin L, Brenner R and Suquet P 2019 Modelling and Simulation in Materials Science and Engineering 27085012 publisher: IOP Publishing

[17] Courant R, Friedrichs K and Lewy H 1928 Mathematische Annalen 100 32-74

[18] Courant R, Friedrichs K and Lewy H 1967 IBM Journal of Research and Development 11 215-234

[19] Hyman J M 1976 The method of lines solution of partial differential equations Ph.D. thesis New York University New York, NY, USA

[20] Di Pietro D A and Ern A 2012 Mathematical Aspects of Discontinuous Galerkin Methods Mathématiques et Applications (Berlin Heidelberg: Springer-Verlag)

[21] Shu C W and Osher S 1988 Journal of Computational Physics 77 439-471

[22] Gottlieb D and Shu C W 1997 SIAM Review 39 644-668

[23] Kuzmin D 2010 Journal of Computational and Applied Mathematics 233 3077-3085

[24] Kuzmin D 2013 International Journal for Numerical Methods in Fluids 71 1178-1190

[25] Ebrahimi A, Monavari M and Hochrainer T 2014 MRS Online Proceedings Library (OPL) 1651

[26] Wagner L 2019 A discontinuous Galerkin method for continuum dislocation dynamics in a fully-coupled elastoplasticity model PhD Thesis Karlsruher Institut für Technologie (KIT)

[27] Schulz K, Wagner L and Wieners C 2019 International Journal of Plasticity 120 248-261

[28] Hochrainer T, Sandfeld S, Zaiser M and Gumbsch P 2014 Journal of the Mechanics and Physics of Solids 63 167-178

[29] Salencon J 2001 Handbook of Continuum Mechanics: General Concepts Thermoelasticity (Berlin Heidelberg: Springer-Verlag)

[30] Burman E, Quarteroni A and Stamm B 2010 Journal of Scientific Computing 43 293-312

[31] Gottlieb S and Shu C W 1998 Mathematics of Computation 67 73-85

[32] Logg A, Mardal K A and Wells G 2012 Automated solution of differential equations by the finite element method: The FEniCS book vol 84 (Springer Science \& Business Media)

[33] Alnæs M, Blechta J, Hake J, Johansson A, Kehlet B, Logg A, Richardson C, Ring J, Rognes M E and Wells G N 2015 Archive of Numerical Software 3

[34] Burman E, Ern A and Fernandes M A 2010 SIAM Journal on Numerical Analysis 48 2019-2042 
[35] Zhang Q and Shu C W 2004 SIAM Journal on Numerical Analysis 42 641-666

[36] Landet T, Mardal K A and Mortensen M 2020 Computers \& Fluids 196104322

[37] Landet T and Mortensen M 2019 arXiv:1903.11943 [physics]

[38] Landet T 2019 Journal of Open Source Software 41239

[39] Cockburn B, Li F and Shu C W 2004 Journal of Computational Physics 194 588-610 